\title{
Distortion inequality for the Frobenius-Perron operator and some of its consequences in ergodic theory of Markov maps in $\mathbb{R}^{d}$
}

\author{
by Piotr Bugiel (Kraków)
}

\begin{abstract}
Asymptotic properties of the sequences
\end{abstract}
(a) $\left\{P_{\varphi}^{j} g\right\}_{j=1}^{\infty}$ and

(b) $\left\{j^{-1} \sum_{i=0}^{j-1} P_{\varphi}^{i} g\right\}_{j=1}^{\infty}$,

where $P_{\varphi}: L^{1} \rightarrow L^{1}$ is the Frobenius-Perron operator associated with a nonsingular Markov map defined on a $\sigma$-finite measure space, are studied for $g \in G=\left\{f \in L^{1}: f \geq 0\right.$ and $\|f\|=1\}$. An operator-theoretic analogue of Rényi's Condition is introduced. It is proved that under some additional assumptions this condition implies the $L^{1}$-convergence of the sequences (a) and (b) to a unique $g_{0} \in G$. The general result is applied to some smooth Markov maps in $\mathbb{R}^{d}$. Also the Bernoulli property is proved for a class of smooth Markov maps in $\mathbb{R}^{d}$.

1. Introduction. The so-called Rényi's Condition occurred explicitly for the first time in [Re57] (called there "Condition C"). Rényi deduced from it existence and ergodicity of invariant measures for a broad class of piecewise monotonic transformations of the unit interval $[0,1]$ into itself.

Somewhat later Rokhlin proved under that condition that some of the number-theoretic transformations studied by Rényi have a much stronger property than ergodicity, namely, they are exact endomorphisms, (a concept introduced by himself) [Ro61]. Since then the condition has rapidly become to play a very useful role in ergodic theory of point transformations [Sch89].

On the other hand, an important role in the study of ergodic properties of point transformations is played by the so-called Frobenius-Perron operator (known also as the transfer operator, or Kuzmin operator) [R56, Sch89, U60]. This is due to the fact that there are close connections between several

1991 Mathematics Subject Classification: 28D05, 58F08, 58F15, 47A50.

Key words and phrases: invariant measure, Frobenius-Perron operator, expanding map, distortion inequality. 
ergodic properties of transformations and the associated Frobenius-Perron operator.

For example, a point transformation $\varphi$ and the corresponding FrobeniusPerron operator $P_{\varphi}$ satisfy the following relation: there exists a unique $g_{0} \in G=\left\{f \in L^{1}: f \geq 0\right.$ and $\left.\|f\|=1\right\}$ such that $P_{\varphi} g_{0}=g_{0}$ and $g_{0}=\lim _{j \rightarrow \infty} P_{\varphi}^{j} g$ in $L^{1}$ for all $g \in G \Leftrightarrow \varphi$ is an exact endomorphism over $\left(I, \Sigma, \mu_{0}\right)$, where $d \mu_{0}=g_{0} d m[\operatorname{Li} 71]$.

It turns out that one can also formulate a property of the FrobeniusPerron operator that is connected with Rényi's Condition. Namely, in Section 3 we introduce condition (3.H1) which is an operator-theoretic analogue of Rényi's Condition, and under this condition we study the convergence, on $G$, of the following two sequences: (a) $\left\{P_{\varphi}^{j}\right\}_{j=1}^{\infty}$ and (b) $\left\{j^{-1} \sum_{i=0}^{j-1} P_{\varphi}^{i}\right\}_{j=1}^{\infty}$, where $\varphi$ is a Markov map defined on a $\sigma$-finite measure space $(I, \Sigma, m)$ (see Definitions 2.1 and 2.2).

Notice that if the convergence holds on $G$ in either case (a) or (b), then $\varphi$ necessarily has an absolutely continuous invariant measure. Unfortunately, Rényi's Condition does not generally ensure the existence of such a measure [Bu85, Bu87]. It must be completed to ensure the recurrence property of the Markov maps considered. Accordingly, our operator-theoretic analogue of Rényi's Condition must also be completed.

We give two such complementary conditions: (3.H2) and (3.H3) in Theorems 3.1 and 3.2, respectively. The first condition controls possible tendencies of the mass to escape to a fixed point or to infinity (in the case $m(I)=\infty)$ under the action of $P_{\varphi}^{j}, j=1,2, \ldots$, and the second ensures weak compactness of the sequence of the iterates of $P_{\varphi}$. As a result, (3.H1) together with (3.H2) implies the convergence of $\left\{P_{\varphi}^{j}\right\}_{j=1}^{\infty}$, while together with (3.H3) implies the convergence of $\left\{j^{-1} \sum_{i=0}^{j-1} P_{\varphi}^{i}\right\}_{j=1}^{\infty}$ (see Theorems 3.1 and 3.2 , respectively). Both conditions (3.H2) and (3.H3) are optimally adjusted to (3.H1) in the sense explained in Section 3. Moreover, (3.H2) completes Rényi's Condition and its operator-theoretic analogue in a very effective way (see Examples 4.3.1, 4.3.2, and Remark 4.3.1).

To illustrate the generality and usefulness of the result of Section 3, Theorems 3.1 and 3.2 are applied in Section 4 to some smooth Markov maps in $\mathbb{R}^{d}$. The proofs of the theorems of Section 4 reveal that several combinations of already known conditions imply (3.H1). This makes it possible to derive, in a uniform way, many separate results from two general ones: Theorems 3.1 and 3.2, and thereby to unify them. Assuming smoothness of the transformations considered, one additionally gets smoothness of their invariant densities.

In Section 5 we prove the Bernoulli property for some class of $C^{1+\alpha}$ Markov maps satisfying Rényi's Condition (Theorem 5.1 and Corollary 5.1). 
This extends the result of [Bu93]. In Section 6 we discuss some recently published special cases connected with the theorems of Section 4 .

2. Basic definitions and notations. Let $(I, \Sigma, m)$ be a $\sigma$-finite atomless (nonnegative) measure space. Quite often the notions or relations occurring in this paper (in particular, the transformations considered) are defined or hold only up to sets of $m$-measure zero. Henceforth we do not mention this explicitly.

The restriction of a mapping $\tau: X \rightarrow Y$ to a subset $A \subseteq X$ is denoted by $\tau_{\mid A}$ and the indicator function of a set $A$ by $\mathbf{1}_{A}$.

Let $\tau: I \rightarrow I$ be a measurable transformation, i.e. $\tau^{-1}(A) \in \Sigma$ for each $A \in \Sigma$. It is called nonsingular iff $m \circ \tau^{-1} \sim m$, i.e. for each $A \in \Sigma$, $m\left(\tau^{-1}(A)\right)=0 \Leftrightarrow m(A)=0$.

Definition 2.1. A nonsingular transformation $\varphi$ from $I$ into itself is said to be piecewise invertible iff

(2.M1) one can find a finite or countable partition $\pi=\left\{I_{k}: k \in K\right\}$ of $I$ into measurable subsets such that $m\left(I_{k}\right)>0$ for each $k \in K$, and $\sup \left\{m\left(I_{k}\right): k \in K\right\}<\infty$;

(2.M2) for each $I_{k} \in \pi$, the mapping $\varphi_{k}=\varphi_{\mid I_{k}}$ is one-to-one from $I_{k}$ onto $J_{k}=\varphi_{k}\left(I_{k}\right)$ and its inverse $\varphi_{k}^{-1}$ is measurable.

Several important classes of piecewise invertible transformations, e.g. Anosov diffeomorphisms [Ma87], some expanding mappings [Sz84], or unimodal mappings [MS93] admit partitions with the so-called Markov property. In this paper we restrict ourselves to the study of such piecewise invertible transformations:

Definition 2.2. A piecewise invertible transformation $\varphi$ is said to be a Markov map iff the corresponding partition $\pi$ satisfies the following two conditions:

(2.M3) $\quad \pi$ is a Markov partition, i.e. for each $k \in K, \varphi\left(I_{k}\right)=\bigcup\left\{I_{j}\right.$ : $\left.m\left(\varphi\left(I_{k}\right) \cap I_{j}\right)>0\right\}$

(2.M4) $\varphi$ is indecomposable (irreducible) with respect to $\pi$, i.e. for each $k \in K, \bigcup_{n=1}^{\infty} \varphi^{n}\left(I_{k}\right)=I$.

In what follows we denote by $\|\cdot\|$ the norm in $L^{1}=L^{1}(I, \Sigma, m)$ and by $G=G(m)$ the set of all (probability) densities, i.e.

$$
G:=\left\{g \in L^{1}: g \geq 0 \text { and }\|g\|=1\right\} .
$$

Let $\tau: I \rightarrow I$ be a nonsingular transformation. Then the formula

$$
P_{\tau} f:=\frac{d}{d m}\left(m_{f} \circ \tau^{-1}\right) \quad \text { for } f \in L^{1},
$$


where $d m_{f}=f d m$, and $d / d m$ denotes the Radon-Nikodym derivative, defines a linear operator from $L^{1}$ into itself. It is called the Frobenius-Perron operator (F-P operator, for short) associated with $\tau$ [R56, U60].

The operator $P_{\tau}$ is a contraction, i.e. $\left\|P_{\tau}\right\| \leq 1$; moreover, $P_{\tau} G \subseteq G$, and $P_{\tau} g=g$ (for some $g \in G$ ) if and only if the measure $d \mu=g d m$ is $\tau$-invariant, i.e. $\mu \circ \tau^{-1}=\mu$; also,

$$
P_{\tau}^{j}=P_{j}, \quad \text { where } P_{j} \text { is the F-P operator associated with } \tau^{j} .
$$

A measure-preserving transformation $\tau: I \rightarrow I$ (i.e., there exists a $\tau$ invariant measure $\mu$ ) is called an exact endomorphism iff $\bigcap_{j=0}^{\infty} \tau^{-j}(\Sigma)=$ $\{\emptyset, I\}(\bmod \mu)[\operatorname{Ro} 61]$.

The exactness of an endomorphism $\tau$ is equivalent to the following property of its F-P operator $P_{\tau}$ [Li71]: there exists a density $\widetilde{g} \in G$ such that

$$
\widetilde{g}=\lim _{j \rightarrow \infty} P_{\tau}^{j} g \quad \text { for all } g \in G .
$$

The following criterion of exactness of $\tau$ is used in the proof of Theorem 3.1 (see [LY82, Th. 2, and Rem. 1]):

TheOREM 2.1. Suppose there exist $h \in L^{1}, h \geq 0$ with $\|h\|>0$, and a dense subset $G_{0} \subseteq G$ such that $\lim _{j \rightarrow \infty}\left\|\left(P_{\tau}^{j} g-h\right)^{-}\right\|=0$ for all $g \in G_{0}$. Then there is a unique $\widetilde{g} \in G$ such that $\left(2.2^{*}\right)$ holds.

For $r \geq 1$, let $\varphi^{r}$ be the $r$ th iterate of a Markov map $\varphi$. Then $\varphi^{r}$ is a Markov map with Markov partition

$$
\pi^{r}:=\bigvee_{j=0}^{r-1} \varphi^{-j}(\pi), \quad \pi^{1}=\pi
$$

Next, for any multi-index $k(r)=\left(k_{0}, k_{1}, \ldots, k_{r-1}\right) \in K^{r}$ we define

$$
\begin{gathered}
\varphi_{k(r)}:=\left(\varphi^{r}\right)_{\mid I_{k(r)}} \text { and } J_{k(r)}:=\varphi_{k(r)}\left(I_{k(r)}\right), \quad \text { where } \\
I_{k(r)}:=I_{k_{0}} \cap \varphi^{-1}\left(I_{k_{1}}\right) \cap \ldots \cap \varphi^{-(r-1)}\left(I_{k_{r-1}}\right) \in \pi^{r} .
\end{gathered}
$$

Clearly, $\varphi_{k(r)}$ is one-to-one from $I_{k(r)}$ onto $J_{k(r)}:=\varphi_{k(r)}\left(I_{k(r)}\right)=\varphi\left(I_{k_{r-1}}\right)$. It is nonsingular, and $\varphi_{k(r)}^{-1}$ is also measurable.

It follows that the formula

$$
m_{k(r)}(A):=m\left(\varphi_{k(r)}^{-1}(A)\right) \quad \text { for } A \in \Sigma
$$

defines an absolutely continuous measure which is concentrated on $J_{k(r)}$ (i.e., $m_{k(r)}(A)=m_{k(r)}\left(A \cap J_{k(r)}\right)$ ), and whose Radon-Nikodym derivative satisfies $d m_{k(r)} / d m>0$ a.e. on $J_{k(r)}$. 
To see the latter property, note first that if $d m_{k(r)} / d m=0$ on $A \subseteq J_{k(r)}$, then $\varphi_{k(r)}^{-1}(A) \subseteq I \backslash I_{k(r)}$ a.e., because

$$
m\left(\varphi_{k(r)}^{-1}(A) \cap I_{k(r)}\right)=\int_{A \cap J_{k(r)}} \frac{d m_{k(r)}}{d m} d m=0 .
$$

Therefore, $A=\emptyset$ a.e.

We put (for $r=1,2, \ldots$ )

$$
\begin{aligned}
& \sigma_{k(r)}:= \begin{cases}d m_{k(r)} / d m & \text { on } J_{k(r)}, \\
0 & \text { on } I \backslash J_{k(r)},\end{cases} \\
& \bar{f}_{k(r)}:= \begin{cases}f \circ \varphi_{k(r)}^{-1} & \text { on } J_{k(r)}, \\
0 & \text { on } I \backslash J_{k(r)} .\end{cases}
\end{aligned}
$$

Then the $r$ th iterate $P_{\varphi}^{r}$ of the F-P operator $P_{\varphi}$ (we often write $P$ instead of $P_{\varphi}$ ) can be written in the form

$$
P_{\varphi}^{r} f=\sum_{k(r)} \bar{f}_{k(r)} \sigma_{k(r)} .
$$

Indeed, from (2.1), (2.2), Definition 2.2 and (2.5) it follows that for any $f \in L^{1}, f \geq 0$ the following equalities hold:

$$
\begin{aligned}
\int_{A} P_{\varphi}^{r} f d m & =m_{f}\left(\varphi^{-r}(A)\right)=\sum_{k(r)} \int_{A_{k(r)}} f d m=\sum_{k(r)} \int_{A} f \circ \varphi_{k(r)}^{-1} d m_{k(r)} \\
& =\int_{A}\left(\sum_{k(r)} \bar{f}_{k(r)} \sigma_{k(r)}\right) d m,
\end{aligned}
$$

where $A_{k(r)}=\varphi_{k(r)}^{-1}(A)$. Hence, (2.8) follows.

3. Convergence theorems. It is well known that the Uniformly Expanding Condition and the Second Derivative Condition ((4.2.H8) and (4.3.H9) in this paper) imply that a Markov map $\varphi$ satisfies Rényi's Condition [Re57], i.e. its iterates $\varphi^{r}(r=1,2, \ldots)$ satisfy the so-called distortion inequality. It turns out that under the two above mentioned conditions also the iterates $P_{\varphi}^{r}(r=1,2, \ldots)$ of the Frobenius-Perron operator $P_{\varphi}$ satisfy some kind of distortion inequality on some dense subsets of $G$. This fact was exploited by the author in his studies of ergodic properties of Markov maps (see, e.g., [Bu82a] or [Bu91a, Prop. 2.1]). In this paper we take this property of the Frobenius-Perron operator as the starting point of our considerations. In the next section we show that several combinations of already known conditions imply this property of $P_{\varphi}$. This makes it possible to derive many previously known separate theorems from two general ones: Theorems 3.1 and 3.2 . 
Let $\varphi$ be a Markov map with Markov partition $\pi=\left\{I_{k}: k \in K\right\}$ and let $P_{\varphi}$ be its F-P operator. We put

$$
A_{j}(g):=\sup _{k \in K} \frac{A_{j k}(g)}{a_{j k}(g)},
$$

where

$$
\begin{aligned}
A_{j k}(g) & :=\operatorname{ess} \sup \left\{P_{\varphi}^{j} g(x): x \in I_{k} \cap \operatorname{spt}\left(P_{\varphi}^{j} g\right)\right\}, \\
a_{j k}(g) & :=\operatorname{ess} \inf \left\{P_{\varphi}^{j} g(x): x \in I_{k} \cap \operatorname{spt}\left(P_{\varphi}^{j} g\right)\right\}, \\
\operatorname{spt}(g) & :=\{x: g(x)>0\} .
\end{aligned}
$$

Then for every constant $C^{*}>0$, we put

$$
G^{*}\left(C^{*}\right):=\left\{g \in G: \exists_{r \geq 1} \operatorname{spt}(g) \in \Sigma^{r}, \text { and } \limsup _{j \rightarrow \infty} A_{j}(g) \leq C^{*}\right\},
$$

where $\Sigma^{r}=\sigma\left(\pi^{r}\right)$ is the smallest $\sigma$-algebra generated by the Markov partition $\pi^{r}$ given by (2.3).

The following fact follows from the definition of $G^{*}$ :

FACT 3.1. $G^{*}$ is a convex subset of $G$.

There are two problems connected with $G^{*}$. The first one is its size. Let $G^{*}\left(C^{*}\right) \neq \emptyset$ for some $C^{*}>0$. Then the iterates $P_{\varphi}^{r}(r=1,2, \ldots)$ satisfy the above mentioned distortion inequality on $G^{*}$. For the $L^{1}$-convergence of $\left\{P_{\varphi}^{j} g\right\}_{j=1}^{\infty}$ or $\left\{j^{-1} \sum_{i=0}^{j-1} P_{\varphi}^{i} g\right\}_{j=1}^{\infty}$ for all $g \in G$, it is not enough to assume merely that $G^{*} \neq \emptyset$. Here, one has to assume that $G^{*}$ is dense in $G$. The motivation is as follows: for the former sequence, if $G^{*} \neq \emptyset$, then inequality (3.8) below holds for all $g \in G^{*}$ (without any additional assumptions). It implies the decisive (for the $L^{1}$-convergence) relation (3.9) provided that $G^{*}$ is dense. For the latter sequence the denseness of $G^{*}\left(C^{*}\right)$ is needed to get the convergence on the whole $L^{1}$ by using the Yosida-Kakutani Ergodic Theorem. The assumption that $G^{*}$ is dense therefore plays a central role in what follows:

(3.H1) (Distortion Inequality for $P_{\varphi}$ ) There exists a constant $C^{*}>0$ such that the set $G^{*}\left(C^{*}\right)$ contains a subset dense in $G$.

The second problem is that even when $G^{*}$ is dense, this does not ensure the convergence of the two sequences (see Remark 3.2).

We introduce two additional conditions: (3.H2) and (3.H3), and prove that (3.H1) in conjunction with the first or second condition implies the $L^{1}$ convergence of $\left\{P_{\varphi}^{j} g\right\}_{j=1}^{\infty}$ or $\left\{j^{-1} \sum_{i=0}^{j-1} P_{\varphi}^{i} g\right\}_{j=1}^{\infty}$, respectively, to a unique $P_{\varphi}$-invariant density $g_{0}$, independent of $g$.

The first condition is much less restrictive than the second (see Examples 4.3.1, 4.3.2, and Remark 4.3.1), but the latter is readily verifiable in practice (especially in the case $m(I)<\infty$, see Fact 4.1.1). Moreover, from (3.6), 
(3.8), (3.10) and (3.11) it follows that each of these two conditions completes (3.H1) in an optimal way.

We define

$$
u_{r}(x):=\inf \left\{g_{k(r)}(x): k(r) \in K^{r}, I_{k(r)} \neq \emptyset\right\},
$$

and

$$
U_{r}(x):=\sup \left\{g_{k(r)}(x): k(r) \in K^{r}, I_{k(r)} \neq \emptyset\right\}
$$

where

$$
g_{k(r)}:=\sum_{s(r)} \widetilde{\sigma}_{s(r)} \int_{I_{s(r)}} \tilde{\sigma}_{k(r)} d m,
$$

and

$$
\widetilde{\sigma}_{k(r)}:=\frac{\sigma_{k(r)}}{m\left(I_{k(r)}\right)},
$$

and $I_{k(r)}$ and $\sigma_{k(r)}$ are defined by (2.4) and (2.6), respectively.

Now, let $\left\{V_{n}\right\}_{n=1}^{\infty}$ be a sequence of subsets of $I$ such that each $V_{n}$ is the union of a finite number of $I_{k}$ 's, $V_{n} \subset V_{n+1}, \bigcup_{n=1}^{\infty} V_{n}=\widetilde{I}$ and $m(I \backslash \widetilde{I})=0$. Then we define

$$
d_{r n}:=\sup _{k(r)} \int_{I \backslash V_{n}} \widetilde{\sigma}_{k(r)} d m .
$$

We now prove the basic results of this paper.

Theorem 3.1 (First Convergence Theorem). Let a Markov map $\varphi$ satisfy (3.H1) and the following condition:

(3.H2) there exists $\widetilde{r} \geq 1$ such that $\left\|u_{\widetilde{r}}\right\|>0$.

Then:

(a) There exists exactly one $P_{\varphi}$-invariant density $g_{0}$ such that $\lim _{j \rightarrow \infty} P_{\varphi}^{j} g$ $=g_{0}$ for all $g \in G$; in consequence, the semi-dynamical system $\left(I, \Sigma, d \mu_{0}, \varphi\right)$, where $d \mu_{0}=g_{0} d m$, is exact ( $\varphi$ is an exact endomorphism).

(b) There exists a density of the form

$$
\widetilde{g}_{0}:=\sum_{k(\widetilde{r})} \widetilde{\sigma}_{k(\widetilde{r})} \int_{I_{k(\widetilde{r})}} g_{0} d m
$$

with $\widetilde{r}$ as in (3.H2), such that for any (fixed) $C_{*}>C^{*}$,

$$
\widetilde{g}_{0} / C_{*} \leq g_{0} \leq C_{*} \widetilde{g}_{0} \quad \text { and } \quad \widetilde{g}_{0}>0 .
$$

$\left(\mathrm{b}_{1}\right)$ Additionally, the unique $\varphi$-invariant density $g_{0}$ is estimated as follows:

$$
u_{\widetilde{r}} / C_{*}^{2} \leq g_{0} \leq C_{*}^{2} U_{\widetilde{r}}
$$

where $U_{\widetilde{r}}$ is defined by $\left(3.1^{*}\right)$. The upper estimate holds provided $\left\|U_{\widetilde{r}}\right\|<\infty$. 
Proof. (a) By (3.H1) there exists a subset $G_{*} \subseteq G^{*}$ dense in $G$. Fix $C_{*}>C^{*}$. Then for any $g \in G_{*}$ there exists $j_{1}=j_{1}(g)$ such that for any $j \geq j_{1}$ and all $I_{k} \in \pi$ one has

$$
\frac{1}{C_{*}} \leq \frac{P^{j} g(x)}{P^{j} g(y)} \leq C_{*} \quad \text { for } m \times m \text {-a.e. }(x, y) \in I_{k} \times I_{k} .
$$

This gives the following basic double inequality (see [Bu91a, (3.4)]):

$$
\frac{F_{r}\left(P^{j} g\right)}{C_{*}} \leq P^{r} P^{j} g \leq C_{*} F_{r}\left(P^{j} g\right)
$$

for $g \in G_{*}, r \geq 1$, and $j \geq j_{1}(g)$, where $F_{r}$ is defined by

$$
F_{r}(g):=\sum_{k(r)} \widetilde{\sigma}_{k(r)} \int_{I_{k(r)}} g d m \quad \text { for } g \in G .
$$

Indeed, from (3.5) we obtain

$$
C_{*}^{-1}{\overline{\left(P^{j} g\right)}}_{k(r)}(x) \sigma_{k(r)}(x) \leq{\overline{\left(P^{j} g\right)}}_{k(r)}(y) \sigma_{k(r)}(x) \leq C_{*}{\overline{\left(P^{j} g\right)}}_{k(r)}(x) \sigma_{k(r)}(x)
$$

for each $J_{k(r)}=\varphi_{k(r)}\left(I_{k(r)}\right)$, all $x, y \in J_{k(r)}$, and $j \geq j_{1}(g)$, where

$$
{\overline{\left(P^{j} g\right)}}_{k(r)}(x)= \begin{cases}\left(P^{j} g\right) \circ \varphi_{k(r)}^{-1}(x) & \text { for } x \in J_{k(r)}, \\ 0 & \text { for } x \in I \backslash J_{k(r)} .\end{cases}
$$

Integrating the above inequalities with respect to $x$ on $J_{k(r)}$ and multiplying by $\widetilde{\sigma}_{k(r)}(y)$, then summing the resulting inequalities with respect to all $k(r)$ and finally using the equality $P_{\varphi^{r}} g=P_{\varphi}^{r} g$, one gets (3.6).

Iterating the first inequality of (3.6), using the equality

$$
\sum_{k(r)}\left\|\mathbf{1}_{I_{k(r)}} P^{j} g\right\|=1
$$

and the definition (3.1), one gets for every $r \geq 1$, and all $j \geq j_{1}(g)$,

$$
P^{j+2 r} g \geq \frac{u_{r}}{C_{*}^{2}}
$$

We now show that (3.8) together with (3.H2) implies the existence of a nontrivial lower function for $P$, i.e. a function $h \geq 0$ with $\|h\|>0$ such that $\left\|\left(P^{j} g-h\right)^{-}\right\| \rightarrow 0$ as $j \rightarrow \infty$ for all $g \in G$.

Note first that each $u_{r}$ given by (3.1) is a lower function for $P$. Indeed, since $G_{*}$ is dense in $G$ and $P$ is a contraction, (3.8) implies that, for each $r \geq 1$,

$$
\lim _{j \rightarrow \infty}\left\|\left(P^{j+2 r} g-u_{r}\right)^{-}\right\|=0 \quad \text { for all } g \in G .
$$

Unfortunately, it may happen that $\left\|u_{r}\right\|=0$ for each $r \geq 1$ (see [Bu85, Bu87], and also Counterexample 6.1 below). Now (3.H2) guarantees that $u_{\widetilde{r}}$ is a nontrivial lower function. Finally, the proof of assertion (a) is completed by an appeal to Theorem 2.1 . 
(b) The double inequality follows from (3.6) and (a). Hence for each $A \in$ $\Sigma, \mu_{0}(A)=0 \Leftrightarrow \widetilde{\mu}_{0}(A)=0$ where $d \widetilde{\mu}_{0}=\widetilde{g}_{0} d m$. To prove that $\widetilde{g}_{0}>0$, note first that $J_{k(\widetilde{r})} \subseteq \operatorname{spt}\left(\widetilde{g}_{0}\right)$ for some $k(\widetilde{r})$, because $\left\|\mathbf{1}_{I \sim(\widetilde{r})} g_{0}\right\|>0$ for some $I_{k(\widetilde{r})}$, and $\widetilde{\sigma}_{k(\widetilde{r})}>0$ on $J_{k(\widetilde{r})}$ for each $J_{k(\widetilde{r})}$. Now the equalities $\operatorname{spt}\left(g_{0}\right)=\operatorname{spt}\left(\widetilde{g}_{0}\right)$ and $\varphi\left(\operatorname{spt}\left(g_{0}\right)\right)=\operatorname{spt}\left(g_{0}\right)$ and the indecomposability condition (2.M4) show that $\operatorname{spt}\left(\widetilde{g}_{0}\right)=I$.

$\left(b_{1}\right)$ The estimates follow from (3.6), (3.7) and (a).

Theorem 3.2 (Second Convergence Theorem). Let a Markov map $\varphi$ satisfy (3.H1) and the following condition:

(3.H3) there exists $\widetilde{r} \geq 1$ such that $\lim _{n \rightarrow \infty} d_{\widetilde{r} n}=0$.

Then:

(a) There exists exactly one $P_{\varphi}$-invariant density $g_{0}$ such that $\lim _{j \rightarrow \infty} S_{j} g$ $=g_{0}$ in $L^{1}$ for all $g \in G$, where $S_{j}=j^{-1} \sum_{i=0}^{j-1} P_{\varphi}^{i}$.

(b) Assertions (b) and $\left(\mathrm{b}_{1}\right)$ of the previous theorem hold.

Proof. (a) Let $V_{n}$ be the union of a finite number of $I_{k}$ 's. Then from the right inequality of $(3.5)$ one gets

$$
P^{j} g \leq C_{*} \tilde{m}\left(V_{n}\right)^{-1} \quad m \text {-a.e. on } V_{n},
$$

where $\widetilde{m}\left(V_{n}\right)=\min \left\{m\left(I_{k}\right): I_{k} \subseteq V_{n}\right\}$.

Next, from the right inequality of (3.6) and the definition (3.4) it follows that

$$
\sup _{j \geq j_{1}} \int_{I \backslash V_{n}} P^{j} g d m \leq C_{*} d_{r n} \quad \text { for } r, n=1,2, \ldots
$$

The last two inequalities and (3.H3) imply weak compactness of $\left\{P^{j} g\right\}$ for $g \in G_{*}$ [DS63, Th. IV.8.9]. Assertion (a) now follows from the YosidaKakutani Ergodic Theorem [DS63, Th. VIII.5.1] and the denseness of $G_{*}$ in $G$.

(b) This follows from (a), just as assertions (b) and ( $\left.b_{1}\right)$ of the previous theorem follow from (a) of that theorem.

Remark 3.1. The set $G^{*}$ of (3.H1) may contain several different dense subsets of $G$. This is the case, for instance, under the assumptions of Theorem 4.3.7 below. Indeed, there exists a dense subset $G_{c} \neq \emptyset$ of $G$ because (4.2.H8) and (4.3.H9) imply (4.1.H5), which in turn implies (4.1). This last inclusion together with Fact 4.3 show that $G_{c} \neq \emptyset$ is dense for $c=1$ because $S_{\infty} G \subseteq G$, where $S_{\infty} G$ is defined by $\left(4.1^{*}\right)$.

On the other hand, the class $\widetilde{G}(1)$ given by Definition 4.3 .4 is different from $G_{c}$ and dense in $G$, too. 
REMARK 3.2. We have already mentioned that (3.H1) alone does not ensure in general the $L^{1}$-convergence of $\left\{P_{\varphi}^{j} g\right\}$ and $\left\{S_{j} g\right\}$ to a unique $P_{\varphi^{-}}$ invariant density. It may happen that under this condition neither (3.H2) nor (3.H3) holds. Examples of Markov maps that satisfy (3.H1) (actually, (4.2.H8) and (4.3.H9), which, as remarked at the beginning of this section, imply (3.H1)) and that have no absolutely continuous invariant measure are given in [Bu85, Bu87] (see also Counterexample 6.1). Note that the second iterate $\varphi^{2}$ of these Markov maps already satisfies $\varphi^{2}\left(I_{k}\right)=I$ for each $I_{k}$. Therefore $\left(6 . \mathrm{M}_{2} 4\right)$ holds for the smallest possible $j=2$ (for $j=1$ we get $\left(3 . \mathrm{M}_{1} 4\right)$ below).

REMarK 3.3. Conditions (3.H2), (3.H3) and (4.1.H6) (which is, by Fact 4.1.1, equivalent to (3.H3)) occurred in [Bu82a]. Condition (3.H2) was already announced in $[\mathrm{Bu} 82,(4.8)]$, while $(3 . \mathrm{H} 3)$ was used there in the case $I=\mathbb{R}^{\mathbf{1}}$ (see (3.1) there). All these conditions ensure the recurrence property of the Markov maps $\varphi$ whose Markov partitions $\pi$ are neither finite nor satisfy the following very special case of (2.M4):

$\left(3 . \mathrm{M}_{1} 4\right) \quad$ for each $k \in K, \varphi\left(I_{k}\right)=I$.

However, Examples 4.3.1 and 4.3.2 in the next section show that (3.H2) is the most effective.

4. Some applications to smooth Markov maps in $\mathbb{R}^{d}$. In what follows the following notation will be used: || - the Euclidean norm in $\mathbb{R}^{d} ; I$-a domain in $\mathbb{R}^{d}$, i.e. an open, connected subset of $\mathbb{R}^{d} ; \Sigma$-the $\sigma$-algebra of all Borel-measurable subsets of $I ; m$ - the Lebesgue measure on $\mathbb{R}^{d} ; \operatorname{diam}(A)$ the diameter of the set $A ; D f$ (resp. $D_{j} f$ ) - the derivative (resp. partial derivative) of $f$.

A smooth Markov map $\varphi$ (i.e., either $C^{1+\alpha}, 0<\alpha \leq 1$, or $C^{2}$ ) is a Markov map in the sense of Definition 2.2 such that the partition $\pi$ of $\varphi$ consists of domains, and the restriction $\varphi_{k}$ of $\varphi$ to any $I_{k} \in \pi$ is a $C^{1+\alpha}$ (or $C^{2}$ ) diffeomorphism.

To illustrate the generality and usefulness of Theorems 3.1 and 3.2 we show how they yield some ergodic properties of smooth Markov maps.

4.1. $C^{1+\alpha}$ Markov maps: general case. In ergodic theory of $C^{1+\alpha}$ Markov maps the following condition plays a crucial role (see e.g. [Ma87]):

(4.1.H4) (Local case) Let $\varphi \in C^{1+\alpha}\left(I_{k}\right)$ for each $k \in K$. There exists a constant $C_{10}>0$ such that for $r=1,2, \ldots, k(r) \in K^{r}$ and all $I_{k} \in \pi$,

$\left|\sigma_{k(r)}(x)-\sigma_{k(r)}(y)\right| \leq C_{10} \sigma_{k(r)}(y)|x-y|^{\alpha}$ for all $x, y \in J_{k(r)} \cap I_{k}$, where $\sigma_{k(r)}$ and $J_{k(r)}$ are defined by (2.6) and (2.4), respectively. 
For $m(I)<\infty$, its global version is

(4.1. $\mathrm{H} 4) \quad$ (Global case, $m(I)<\infty)$ Let $\varphi \in C^{1+\alpha}\left(I_{k}\right)$ for each $k \in K$. There exists a constant $\widetilde{C}_{10}>0$ such that for $r=1,2, \ldots$ and $k(r) \in K^{r}$,

$\left|\sigma_{k(r)}(x)-\sigma_{k(r)}(y)\right| \leq \widetilde{C}_{10} \sigma_{k(r)}(y)|x-y|^{\alpha} \quad$ for all $x, y \in J_{k(r)}$.

Note that the above two conditions imply, respectively, local and global versions of the so-called Reniyi's Condition ([Re57] or [Ro61]).

The local Reńyi's Condition reads as follows:

(4.1.H5) (Local case) There exists a constant $C_{* *}>0$ such that for all $k(r) \in K^{r}, r=1,2, \ldots$, and for each $I_{k} \in \pi$,

$$
\sup _{x \in J_{k(r)} \cap I_{k}} \sigma_{k(r)}(x) \leq C_{* *} \inf _{x \in J_{k(r)} \cap I_{k}} \sigma_{k(r)}(x) .
$$

Its global counterpart can be written as follows:

(4.1. $\widetilde{H} 5) \quad($ Global case, $m(I)<\infty)$ There exists a constant $\widetilde{C}_{* *}>0$ such that

$$
\sup _{x \in J_{k(r)}} \sigma_{k(r)}(x) \leq \widetilde{C}_{* *} \inf _{x \in J_{k(r)}} \sigma_{k(r)}(x) \quad \text { for all } k(r) \in K^{r}, r=1,2, \ldots
$$

In our context the two constants are $C_{* *}=C_{10, \alpha}:=1+C_{10} \widetilde{C}_{0}^{\alpha}$ and $\widetilde{C}_{* *}=\widetilde{C}_{10, \alpha}:=1+\widetilde{C}_{10} \widetilde{C}_{0}^{\alpha}$ where $\widetilde{C}_{0}=\sup \left\{\operatorname{diam}\left(I_{k}\right): k \in K\right\}<\infty$.

Note that (4.1. $\widetilde{H} 4)$ implies the following very useful fact:

FACT 4.1.1. If $\varphi$ is defined on $I$ with $m(I)<\infty$ and satisfies (4.1. $\widetilde{\mathrm{H}} 4)$ and

$$
C_{11}:=\inf \left\{m\left(\varphi\left(I_{k}\right)\right): k \in K\right\}>0,
$$

then (3.H3) holds. Moreover, (3.H3) and (4.1.H6) are equivalent.

We recall that $\pi$ being a generator for $\varphi$ means the following $(\sigma(\mathcal{A})$ denotes the smallest $\sigma$-algebra generated by the family $\mathcal{A}$ ):

(Generating condition on $\pi) \sigma\left(\pi^{r}: r \geq 1\right)=\Sigma$, where $\pi^{r}$ is defined by (2.3).

Below we show that (4.1.H4) together with (4.1.H7) implies (3.H1). Then under (3.H2) one gets the assertion of Theorem 3.1.

However, (4.1.H4) makes it possible to prove the convergence of $\left\{P^{j} g\right\}$ and $\left\{S_{j} g\right\}$ not only in $L^{1}$ but also in the topology of uniform convergence (on every $I_{k} \in \pi$ ), and the smoothness $\left(C^{0+\alpha}\right)$ of the unique $P_{\varphi}$-invariant limit density.

The latter fact is proved for the following subset of densities: 
Definition 4.1.3. We denote by $G_{\alpha}, 0<\alpha \leq 1$, the class of all densities $g \in G$ satisfying the following three conditions:

(a) $\operatorname{spt}(g)$ is the union of a number of $I_{k}$ 's;

(b) for each $I_{k} \in \pi, g_{\mid I_{k}} \in C^{0+\alpha}\left(I_{k}\right)$, and

$$
|g(x)-g(y)| \leq C(g) g(y)|x-y|^{\alpha} \quad \text { for all } x, y \in \operatorname{spt}(g) \cap I_{k},
$$

where $C(g)$ is a constant depending on $g$.

The following theorem is a $C^{1+\alpha}$ counterpart of Theorem 3.1:

Theorem 4.1.1. Let a Markov map $\varphi$ satisfy:
(A)
Conditions (3.H2), (4.1.H4), and (4.1.H7) hold.

Then:

$\left(\mathrm{D}_{1}\right) \quad$ Assertions (a), (b) and $\left(\mathrm{b}_{1}\right)$ of Theorem 3.1 hold.

$\left(\mathrm{D}_{2} . \mathrm{c}\right) \quad$ For each $k \in K, \lim _{j \rightarrow \infty}\left\|g_{0}-P_{\varphi}^{j} g\right\|_{k}=0$ for all $g \in G_{\alpha}$ where $\|g\|_{k}=\sup \left\{|g(x)|: x \in I_{k}\right\}$

$\left(\mathrm{D}_{2} . \mathrm{d}\right) \quad\left|g_{0}(x)-g_{0}(y)\right| \leq\left(C_{10} C_{10, \alpha} / m\left(I_{k}\right)\right)|x-y|^{\alpha}$ for $x, y \in I_{k}$.

Proof. $\left(\mathrm{D}_{1}\right)$ For a given constant $c>0$, let $Z_{c, r}$ denote the class of all densities $g \in G$ such that for each $I_{k(r)} \in \pi^{r}$ :

(a) either $g_{\mid I_{k(r)}}>0$ or $=0$;

(b) $g(x) \leq c g(y)$ for $m \times m$-a.e. $(x, y) \in I_{k(r)} \times I_{k(r)}$.

First we note that by (4.1.H5) (which follows from (4.1.H4)),

$$
Z_{c}:=\bigcup_{r=1}^{\infty} \operatorname{conv}\left[Z_{c, r}\right] \subseteq G^{*}\left(C^{*}\right)
$$

where $G^{*}$ is the family of densities defined by (3.0) and $C^{*}=C_{* *} c$.

Now $Z_{1}$ contains

$$
S_{\infty} G:=\bigcup_{r=1}^{\infty} \operatorname{conv}\left[W_{r}\right] \subset G,
$$

where $W_{r}$ is the class of all densities of the form

$$
w_{k(r)}=\mathbf{1}_{I_{k(r)}} / m\left(I_{k(r)}\right), \quad k(r) \in K^{r},
$$

and $\operatorname{conv}\left[W_{r}\right]$ denotes the convex hull of $W_{r}$. Note that $W_{r} \subseteq \operatorname{conv}\left[W_{r+1}\right]$ for $r=1,2, \ldots$.

Since $S_{\infty} G$ is dense in $G$ by (4.1.H7), we get (3.H1). Thus ( $\left.\mathrm{D}_{1}\right)$ holds by Theorem 3.1.

$\left(\mathrm{D}_{2}\right)$ Let $g \in G_{\alpha}$. Then for any $I_{k}$ and $j=1,2, \ldots$,

$$
P^{j} g(x) \leq \frac{C_{\alpha}(g) C_{10, \alpha}}{m\left(I_{k}\right)} \quad \text { for } x \in I_{k},
$$


and

$$
\begin{aligned}
\mid P^{j} g(x)- & P^{j} g(y) \mid \\
& \leq C(g)|x-y|^{\alpha} P^{j} g(x)+C_{10}|x-y|^{\alpha} P^{j} g(y) \\
& \leq\left(C(g) P^{j} g(x)+C_{10} P^{j} g(y)\right)|x-y|^{\alpha} \quad \text { for } x, y \in I_{k},
\end{aligned}
$$

where $C_{\alpha}(g)=1+C(g) \widetilde{C}_{0}^{\alpha}$ and $C_{10, \alpha}=1+C_{10} \widetilde{C}_{0}^{\alpha}$.

The above two inequalities imply that for each $I_{k}$, the family $\left\{P^{j} g\right\}_{j \geq 1}$, restricted to $I_{k}$, is bounded and equicontinuous in the space $C\left(I_{k}\right)$ of all bounded and continuous real functions with the supremum norm.

Thus $\left(\mathrm{D}_{2} . \mathrm{c}\right)$ follows from the Ascoli-Arzelà Lemma and $\left(\mathrm{D}_{1} \cdot \mathrm{a}\right)$.

$\left(\mathrm{D}_{2} . \mathrm{d}\right)$ follows from $\left(\mathrm{D}_{2} . \mathrm{c}\right),(4.2)$ and $(4.3)$, and from the fact that $w_{k}=$ $\mathbf{1}_{I_{k}} / m\left(I_{k}\right) \in G_{\alpha}$ with $C\left(w_{k}\right)=0$.

A $C^{1+\alpha}$ counterpart of Theorem 3.2 is

Theorem 4.1.2. Let a Markov map $\varphi$ satisfy either

(B) (3.H3), (4.1.H4), and (4.1.H7); or

(C) $m(I)<\infty,(4.1 . \widetilde{\mathrm{H}} 4),(4.1 . \mathrm{H} 6)$, and (4.1.H7).

Then:

$\left(\mathrm{D}_{1}\right) \quad$ Assertions (a) and (b) of Theorem 3.2 hold.

$\left(\mathrm{D}_{2} . \mathrm{c}\right) \quad$ For each $k \in K, \lim _{j \rightarrow \infty}\left\|g_{0}-S_{j} g\right\|_{k}=0$ for all $g \in G_{\alpha}$.

$\left(\mathrm{D}_{2} . \mathrm{d}\right) \quad\left|g_{0}(x)-g_{0}(y)\right| \leq\left(C_{10} C_{10, \alpha} / m\left(I_{k}\right)\right)|x-y|^{\alpha}$ for $x, y \in I_{k}$.

Proof. $(B) \Rightarrow\left(D_{1}\right)$. Similarly to the proof of $\left(D_{1}\right)$ of Theorem 4.1.1, the proof is based on the inclusion (4.1).

$(\mathrm{C}) \Rightarrow\left(\mathrm{D}_{1}\right)$. By Fact 4.1 .1 and the previous case.

The two remaining implications are proved analogously.

REMARK 4.1.0. In fact, condition (4.1.H7) is redundant. Namely, it can be derived from (4.1.H5) and the convergence of $\left\{S_{j} g\right\}$ for $g \in G$ (see Proposition 6.1 and Lemma 6.1).

We conclude this subsection with the problem of convergence of $\left\{P^{j} g\right\}$ under (3.H1) and (3.H3). Note that both Theorems 3.2 and 4.1.2 establish in this case only the Cesàro mean convergence.

By Theorems 3.2 and 4.1.2 there exists a unique $\varphi$-invariant measure $d \mu_{0}=g_{0} d m\left(g_{0}>0\right)$. Thus if $\varphi$ is an exact endomorphism over the probability space $\left(I, \Sigma, \mu_{0}\right)$, then, in particular, the following aperiodicity condition is satisfied:

$$
\mu_{0}\left(\varphi^{j}\left(I_{k}\right)\right) \nearrow 1 \quad \text { as } j \rightarrow \infty, \text { for all } k \in K \text {. }
$$

We establish convergence of $\left\{P^{j} g\right\}$ under (3.H1), (3.H3), (4.1.H7), and an additional condition which is close to $\left(4.1 . \mathrm{H}_{0} 3\right)$ and readily checkable in practice (see [Bu91a, $\left.\left(3 . \mathrm{H}_{1} 4\right)\right]$ ): 
(4.1. $\left.\mathrm{H}_{1} 3\right) \quad$ (Aperiodicity condition on $\varphi$ ) There exist an integer $\widetilde{\widetilde{r}}$ and $I_{\widetilde{k}} \in$ $\pi$ such that $\varphi^{\widetilde{r}}\left(I_{\widetilde{k}}\right)=I$.

Note that this condition together with (2.M4) implies that for each $I_{k}$, there exists $j_{1}=j_{1}\left(I_{k}\right)$ such that $\varphi^{j}\left(I_{k}\right)=I$ for $j \geq j_{1}\left(I_{k}\right)$.

We also need a somewhat strengthened version of (4.1.H4):

(4.1. $\left.\mathrm{H}_{1} 4\right) \quad$ (Quasi-global case) Let $\varphi \in C^{1+\alpha}\left(I_{k}\right)$ for each $k \in K$. Then for each $V$ which is the union of a finite number of $I_{k}$ 's, there exists a constant $C_{10}(V)>0$ such that for $r=1,2, \ldots$ and $k(r) \in K^{r}$,

$$
\begin{aligned}
& \left|\sigma_{k(r)}(x)-\sigma_{k(r)}(y)\right| \leq C_{10}(V) \sigma_{k(r)}(y)|x-y|^{\alpha} \\
& \text { for all } x, y \in J_{k(r)} \cap V .
\end{aligned}
$$

Moreover, $C_{10}:=\sup _{k \in K} C_{10}\left(I_{k}\right)<\infty$, i.e., (4.1.H4) holds.

The quasi-global version of Rényi's Condition corresponding to $\left(4.1 . \mathrm{H}_{1} 4\right)$ reads as follows:

(4.1. $\left.\mathrm{H}_{1} 5\right) \quad$ (Quasi-global case) For each $V$ there exists a positive constant $C_{* *}(V)$ such that for all $k(r) \in K^{r}, r=1,2, \ldots$ and for each $V$,

$$
\sup _{x \in J_{k(r)} \cap V} \sigma_{k(r)}(x) \leq C_{* *}(V) \inf _{x \in J_{k(r)} \cap V} \sigma_{k(r)}(x) .
$$

Here $C_{* *}(V)=C_{10, \alpha}(V):=1+C_{10}(V) \widetilde{C}_{0}^{\alpha}$, and $\widetilde{C}_{0}=\sup \left\{\operatorname{diam}\left(I_{k}\right)\right.$ : $k \in K\}<\infty$, in particular $C_{* *}\left(I_{k}\right) \leq C_{* *}$ for $k \in K$ (i.e., (4.1.H5) holds).

The following is a generalization of Proposition 3.1 of [Bu91a]:

THEOREM 4.1.3. Let a Markov map $\varphi$ satisfy either

$\left(\mathrm{B}_{1}\right) \quad$ (3.H3), (4.1.H $\left.H_{1} 3\right),\left(4.1 . H_{1} 4\right)$, and (4.1.H7); or

$\left(\mathrm{C}_{1}\right) \quad m(I)<\infty,\left(4.1 . H_{1} 3\right),(4.1 . \widetilde{H} 4),(4.1 . \mathrm{H} 6)$, and (4.1.H7).

Then the conclusion of Theorem 4.1.1 holds.

Pro of. By Theorem 4.1.2, condition (3.H1) of Theorem 3.1 is satisfied. Thus it remains to show that also (3.H2) holds. A proof that $\left\|u_{s}\right\|>0$ for some $s \geq \widetilde{r}$ is given in [Bu96]. Nevertheless we present it here for the sake of completeness.

The proof proceeds in five steps.

STEP 4.1.1. Let $V$ be the union of a finite number of $I_{k}$ 's. Put $K(V):=$ $\left\{k \in K: I_{k} \subseteq V\right\}$. There exists an integer $\widetilde{s}=\widetilde{s}(V)$ such that for any (fixed) $A \subseteq V, A \in \pi$, and each $j \in K(V)$ there exist $k_{1}(j, A), \ldots, k_{\widetilde{s}-1}(j, A)$ such that

$$
A \subseteq \varphi_{k_{j}(\widetilde{s})}\left(I_{k_{j}(\widetilde{s})}\right), \quad \text { where } \quad k_{j}(\widetilde{s})=\left(j, k_{1}(j, A), \ldots, k_{\widetilde{s}-1}(j, A)\right) .
$$

The proof can be found in [Bu91a, Prop. 3.1]. 
STEP 4.1.2. $d_{r n} \leq C_{* *} d_{\widetilde{r} n}$ for $r \geq \widetilde{r}, n=1,2, \ldots$, where $d_{r n}$ is defined by (3.4).

This follows from the inequality $\widetilde{\sigma}_{(n(s), k(r))} \leq C_{* *} \widetilde{\sigma}_{k(r)}$ for any $s, r \geq 1$, and $n(s) \in K^{s}, k(r) \in K^{r}$.

SteP 4.1.3. For $V$ as in the first step and all $k(r) \in K^{r}, r=1,2, \ldots$,

$$
\begin{aligned}
\frac{1}{m(V) C_{* *}(V)} \mathbf{1}_{J_{k(r)} \cap V} \int_{J_{k(r)} \cap V} \widetilde{\sigma}_{k(r)} d m & \leq \widetilde{\sigma}_{k(r)} \mathbf{1}_{J_{k(r)} \cap V} \\
& \leq \frac{C_{* *}(V)}{\widetilde{m}(V)} \mathbf{1}_{J_{k(r)} \cap V} \quad \text { on } I,
\end{aligned}
$$

where $\widetilde{m}(V)=\min \left\{m\left(I_{k}\right): k \in K(V)\right\}$.

This follows from $\left(4.1 \mathrm{H}_{1} 5\right)$.

STEP 4.1.4. Given $0<\varepsilon<1$, there exists $V$ which is the union of a finite number of $I_{k}$ 's and an integer $s \geq \widetilde{r}$ such that every $A \subseteq V, A \in \pi$ satisfies the following two conditions:

(a) $A \subseteq \bigcap\left\{\varphi_{k_{j}(s)}\left(I_{k_{j}(s)}\right): j \in K(V)\right\}$;

(b) for each $J_{k(s)}$ there exists $j \in K(V)$ such that $m\left(J_{k(s)} \cap I_{k_{j}(s)}\right)>0$, where $k_{j}(s)=\left(j, k_{1}(j, A), \ldots, k_{s-1}(j, A)\right)$.

Indeed, by (3.H3) and Step 4.1.2, there exists a set $V:=V_{\widetilde{n}}, \widetilde{n}=\widetilde{n}(\varepsilon)$, such that

$$
\int_{J_{i(r)} \cap V} \tilde{\sigma}_{i(r)} d m \geq 1-\varepsilon>0 \quad \text { for any } r \geq \widetilde{r} \text { and } i(r) \in K^{r} .
$$

From this and the second inequality of Step 4.1.3 it follows that

$$
m\left(J_{i(r)} \cap V\right) \geq \frac{(1-\varepsilon) \widetilde{m}(V)}{C_{* *}(V)}>0 \quad \text { for any } r \geq \widetilde{r} \text { and } i(r) \in K^{r} .
$$

Thus for $r \geq \widetilde{r}$ and each $J_{i(r)}$ there exists $j \in K(V)$ such that $m\left(J_{i(r)} \cap I_{j}\right)$ $>0$, thus also $I_{j} \subseteq J_{i(r)}$. But $I_{\left(j, j_{1}, \ldots, j_{r-1}\right)} \subset I_{j}$ for all $j_{1}, \ldots, j_{r-1} \in K$, therefore Step 4.1.1 shows that the assertion of Step 4.1.4 holds for $s=$ $\max \{\widetilde{s}, \widetilde{r}\}$.

In the last step we show that the family $\left\{g_{i(s)}\right\}$ of densities, defined by (3.2), is uniformly bounded from below:

STEP 4.1.5.

$$
g_{i(s)} \geq\left(\frac{1-\varepsilon}{m(V) C_{* *}(V)}\right)^{2} m_{s} \mathbf{1}_{V} \quad \text { for each } i(s) \in K^{s},
$$

where $m_{s}=m_{s}(V)>0$ is a constant. 
Indeed, from the first inequality of Step 4.1.3 and $\left(4.3^{*}\right)$ it follows that for each $J_{i(s)}$,

$$
\widetilde{\sigma}_{i(s)} \mathbf{1}_{J_{i(s)} \cap V} \geq \frac{1-\varepsilon}{m(V) C_{* *}(V)} \mathbf{1}_{J_{i(s)} \cap V} .
$$

The inclusion $A \subseteq V$ and Step 4.1.4(a) now show that

$$
\begin{aligned}
g_{i(s)} & \geq \sum_{j \in K(V)} \widetilde{\sigma}_{k_{j}(s)} \mathbf{1}_{J_{k_{j}(s)} \cap V} \int_{I_{k_{j}(s)}} \widetilde{\sigma}_{i(s)} d m \\
& \geq \frac{1-\varepsilon}{m(V) C_{* *}(V)} \mathbf{1}_{A} \int_{A_{1}} \mathbf{1}_{J_{i(s)}} \widetilde{\sigma}_{i(s)} d m,
\end{aligned}
$$

where $A_{1}=\bigcup_{j \in K(V)} I_{k_{j}(s)}$. Since, by Step 4.1.4(b) and $\left(4.3^{* *}\right)$, we have

$$
\inf _{J_{i(s)} \cap A_{1}} \widetilde{\sigma}_{i(s)} \geq \inf _{J_{i(s)} \cap V} \widetilde{\sigma}_{i(s)} \geq \frac{1-\varepsilon}{m(V) C_{* *}(V)},
$$

invoking once more Step 4.1.4(b) yields

$$
g_{i(s)} \geq\left(\frac{1-\varepsilon}{m(V) C_{* *}(V)}\right)^{2} m_{s}(V, A) \mathbf{1}_{A} \quad \text { for each } i(s) \in K^{s},
$$

where $m_{s}(V, A):=\min \left\{m\left(I_{k_{j}(s)}\right): j \in K(V)\right\}$. Thus the inequality of Step 4.1.5 holds with $m_{s}(V):=\min \left\{m_{s}(V, A): A \subseteq V\right\}$.

Together with Theorem 3.1, this finishes the proof of the theorem.

4.2. $C^{1+\alpha}$ Markov maps: Case (I). In some situations Condition (4.1.H4) (or its global version (4.1. $\widetilde{H} 4)$ ) and its consequence, Rényi's Condition (resp., Global Rényi's Condition), follow immediately from the Koebe Principle ([G69]). This is the case, e.g., if the Schwarzian derivative of the Markov map $\varphi$ is negative. This topic is not discussed here.

There are, however, another two important cases strictly connected with those covered by Theorems 4.1.1-4.1.3, and we briefly discuss them next. These are:

(I) $\varphi$ satisfies the Expanding Condition and (4.1.H4), restricted to $r=1$; and

(II) $\varphi$ satisfies the Expanding Condition and the Second Derivative Condition.

Below we sketch how (4.1.H4) can be derived from the hypotheses of Case (I). First however, one has to formulate the Expanding Condition:

(4.2.H8) (Uniformly Expanding (in All Directions) Condition) There exists a constant $C_{1}>1$ such that for each $x \in \widetilde{I}=\bigcup_{k \in K} I_{k}$ the derivative matrix $D \varphi(x)$ satisfies

$$
|D \varphi(x) v| \geq C_{1}|v| \quad \text { for each } v \in \mathbb{R}^{d} .
$$


The discussion of Case (I) needs some facts which are rather well known (see, e.g., [Ma87]). Therefore we restrict ourselves to a convenient formulation:

FACT 4.2.2 (Case (I)). Let a Markov map $\varphi$ satisfy (4.1.H4), or $\left(4.1 . \mathrm{H}_{1} 4\right)$ restricted to $r=1$ (resp., (4.1. $\widetilde{\mathrm{H}} 4$ ) restricted to $r=1$, if $m(I)<\infty$ ) with constants $C_{2, \alpha}>0$ and $C_{2, \alpha}(V)>0$, respectively (resp. $\widetilde{C}_{2, \alpha}>0$ ), and Condition (4.2.H8). Then:

(i) For $r=1,2, \ldots$, and $k(r) \in K^{r}$,

$$
\frac{\sigma_{k(r)}(x)}{\sigma_{k(r)}(y)} \leq \exp \left(C_{5, \alpha}(A)|x-y|^{\alpha}\right) \quad \text { for any } A \text { and } x, y \in J_{k(r)} \cap A,
$$

$\left(\right.$ resp., $\frac{\sigma_{k(r)}(x)}{\sigma_{k(r)}(y)} \leq \exp \left(\widetilde{C}_{5, \alpha}|x-y|^{\alpha}\right) \quad$ for any $\left.x, y \in J_{k(r)}\right)$,

where $A=I_{k}$ or $V$ according as (4.1.H4) or $\left(4.1 . \mathrm{H}_{1} 4\right)$ restricted to $r=1$ is satisfied; and accordingly $C(A)=C_{5, \alpha}:=C_{2, \alpha} C_{1}^{\alpha} /\left(C_{1}^{\alpha}-1\right)$ or $C_{5, \alpha}(V):=$ $C_{2, \alpha}(V) C_{1}^{\alpha} /\left(C_{1}^{\alpha}-1\right)\left(\right.$ resp., $\left.\widetilde{C}_{5, \alpha}:=\widetilde{C}_{2, \alpha} C_{1}^{\alpha}\left(C_{1}^{\alpha}-1\right)\right)$.

(ii) Conditions (4.1.H5), (4.1. $\left.\mathrm{H}_{1} 5\right)$, and (4.1. $\left.\mathrm{H} 5\right)$ hold with $C_{* *}=C_{6, \alpha}$ $:=\exp \left(\widetilde{C}_{0}^{\alpha} C_{5, \alpha}\right), C_{* *}(V)=C_{6, \alpha}(V):=\exp \left(\widetilde{C}_{0}^{\alpha} C_{5, \alpha}(V)\right)$ and $\widetilde{C}_{* *}=C_{9, \alpha}:=$ $\exp \left(\operatorname{diam}(I)^{\alpha} \widetilde{C}_{5, \alpha}\right)$.

(iii) Conditions (4.1.H4) and $\left(4.1 . \mathrm{H}_{1} 4\right)$ hold with $C_{10}:=\max \left\{C_{10}^{\prime}, C_{10}^{\prime \prime}\right\}$ $\times C_{5, \alpha}$, and $C_{10}(V):=\max \left\{C_{10}^{\prime}(V), C_{10}^{\prime \prime}(V)\right\} C_{5, \alpha}(V)$ where $C_{10}^{\prime}, C_{10}^{\prime \prime}$, $C_{10}^{\prime}(V)$, and $C_{10}^{\prime \prime}(V)$ are constants such that $\exp x \leq 1+C_{10}^{\prime} x$ and $1 /(1+$ $\left.C_{10}^{\prime} x\right) \geq 1-C_{10}^{\prime \prime} x$ for $0 \leq x \leq \widetilde{C}_{0}^{\alpha} C_{5, \alpha}$, and $\exp x \leq 1+C_{10}^{\prime}(V) x$ and $1 /\left(1+C_{10}^{\prime}(V) x\right) \geq 1-C_{10}^{\prime \prime}(V) x$ for $0 \leq x \leq \widetilde{C}_{0}^{\alpha} C_{5, \alpha}(V)$, respectively.

Throughout the remainder of this section it is assumed that the domains $J_{k}=\varphi_{k}\left(I_{k}\right), k \in K$, satisfy the following condition:

$\left(4.2 . \mathrm{M}_{1} 2\right) \quad$ There is a constant $C_{0}>0$ such that any two points $x, y$ in any $J_{k}=\varphi_{k}\left(I_{k}\right)$ can be joined by a piecewise straight arc of length at most $C_{0}|x-y|$.

The fact below states that $\pi$ is a generator for $\varphi$ under the following conditions (see [Bu92, Lem. 2.1(b).]):

FACT 4.2.3. Let $\varphi$ satisfy (4.2.H8) and (4.2. $\left.\mathrm{M}_{1} 2\right)$. Then (4.1.H7) holds; more precisely: $\operatorname{diam}\left(I_{k(r)}\right) \leq C_{0} \widetilde{C}_{0} / C_{1}^{r-1}$ for $r=1,2, \ldots$

By using Facts 4.1.1, 4.2.2, and 4.2.3 one can derive from each of Theorems 4.1.1-4.1.3 its counterpart in Case (I). The first of these is

TheOREM 4.2.4. Let a Markov map $\varphi$ satisfy

(A) (3.H2), (4.1.H4) restricted to $r=1$, and (4.2.H8).

Then the conclusion of Theorem 4.1.1 holds. 
P r o o f. Conditions (4.2. $\mathrm{M}_{1} 2$ ) and (4.2.H8) imply (4.1.H7), by Fact 4.2.3. Further, (4.1.H4) restricted to $r=1$ and (4.2.H8) imply (4.1.H4), by Fact 4.2.2. Thus (A) of Theorem 4.1.1 is satisfied.

A counterpart of Theorem 4.1.2 is

TheOREM 4.2.5. Let a Markov map $\varphi$ satisfy either

(B) (3.H3), (4.1.H4) restricted to $r=1$, and (4.2.H8); or

(C) $\quad m(I)<\infty,(4.1 . \widetilde{\mathrm{H}} 4)$, restricted to $r=1$, (4.1.H6), and (4.2.H8).

Then the conclusion of Theorem 4.1.2 holds.

P r o o f. Conditions (4.2. $\mathrm{M}_{1} 2$ ) and (4.2.H8) imply (4.1.H7), by Fact 4.2.3. Further, (4.1.H4) restricted to $r=1$ and (4.2.H8) imply (4.1.H4), in view of Fact 4.2.2. Thus (B) of Theorem 4.1.2 is satisfied.

Also, (C) of Theorem 4.1.2 is satisfied because (4.1. $\widetilde{\mathrm{H}} 4)$ restricted to $r=1$ together with (4.2.H8) implies (4.1. $\widetilde{H} 4)$.

Finally, a counterpart of Theorem 4.1.3 is

TheOREM 4.2.6. Let a Markov map $\varphi$ satisfy either

$\left(\mathrm{B}_{1}\right) \quad$ (3.H3), (4.1. $\left.\mathrm{H}_{1} 3\right),\left(4.1 . \mathrm{H}_{1} 4\right)$ restricted to $r=1$, and (4.2.H8); or

$\left(\mathrm{C}_{1}\right) \quad m(I)<\infty,\left(4.1 . \mathrm{H}_{1} 3\right),(4.1 . \widetilde{\mathrm{H}} 4)$ restricted to $r=1$, and (4.1.H6).

Then the conclusion of Theorem 4.1.1 holds.

P r o o f. Conditions (4.2. $\mathrm{M}_{1} 2$ ) and (4.2.H8) imply (4.1.H7), by Fact 4.2.3. Further, $\left(4.1 . \mathrm{H}_{1} 4\right)$ restricted to $r=1$ and (4.2.H8) imply (4.1. $\left.\mathrm{H}_{1} 4\right)$, by Fact 4.2.2. Thus $\left(\mathrm{B}_{1}\right)$ of Theorem 4.1.3 is satisfied.

Also, $\left(\mathrm{C}_{1}\right)$ of Theorem 4.1.3 is satisfied because (4.1. $\left.\widetilde{\mathrm{H}} 4\right)$ restricted to $r=1$ together with (4.2.H8) implies (4.1. $\widetilde{\mathrm{H}} 4)$.

4.3. $C^{2}$ Markov maps: Case (II). We now turn to Case (II). Before we formulate the Second Derivative Condition we introduce a useful notion, the regularity of a function. Namely, for a given function $f: Y \rightarrow \mathbb{R}$, its regularity $\operatorname{Reg}(f)$ is defined by

$$
\operatorname{Reg}(f):=\sup \left\{\frac{|D f(x)|}{|f(x)|}: x \in Y,|f(x)|>0, D f(x) \text { exists }\right\} .
$$

The Second Derivative Condition can now be formulated as follows:

(4.3.H9) (Second Derivative Condition) For each $k \in K, \varphi \in C^{2}\left(I_{k}\right)$; and $C_{2}:=\sup \left\{\operatorname{Reg}\left(\sigma_{k}\right): k \in K\right\}<\infty$, where $\sigma_{k}$ is defined by (2.6).

Note that instead of (4.3.H9) one uses sometimes, in the 1-dimensional case, the following version ([Bow79]):

(4.3. $\mathrm{H} 9) \quad$ (Second Derivative Condition) For each $k \in K, \varphi \in C^{2}\left(I_{k}\right)$; and $\widetilde{C}_{2}:=\sup \left\{\left|D^{2} \varphi(x)\right| /(D \varphi(y))^{2}: x, y \in I_{k}, k=1,2, \ldots\right\}<\infty$. 
This is justifiable because these two conditions are equivalent ([A79]).

The following class of densities plays an important role in our discussion of ergodic properties of Markov maps in Case (II) (see [Bu91a, Defs. 2.32.4]):

Definition 4.3.4. We denote by $\widetilde{G}(1)$ the class of all densities $g \in G$ satisfying the following four conditions:

(a) $\operatorname{spt}(g)$ is the union of a number of $I_{k}$ 's;

(b) for each $I_{k} \in \pi, g_{\mid I_{k}} \in C^{1}\left(I_{k}\right)$;

(c) $\operatorname{Reg}(g)<\infty$;

(d) $\sup \left\{g(x): x \in I_{k}\right\}<\infty$ for each $I_{k} \in \pi$.

Now we show that some of the results of [Bu91a] follow from Theorem 4.1.1:

Theorem 4.3.7 ([Bu91a, Th. 3.1]). Let a Markov map $\varphi$ satisfy

(A) (3.H2), (4.2.H8), and (4.3.H9).

Then the conclusion of Theorem 4.1.1 holds. In particular, assertion $\left(\mathrm{D}_{2} . \mathrm{c}\right)$ of that theorem holds for densities in $\widetilde{G}(1) \subseteq G_{1}$.

Proof. Under (4.2.H8) and (4.3.H9) the class $\widetilde{G}(1)$ satisfies all the requirements of (3.H1). Indeed, it is dense in $G$ and, by Proposition 2.1 of [Bu91a], $\widetilde{G}(1) \subseteq G^{*}$ for $C^{*}=\exp \left(C_{0} \widetilde{C}_{0} C_{5}\right)$ with any fixed $C_{5}>C_{1} C_{2} /\left(C_{1}\right.$ $-1)$. Additionally, its pleasant feature is $P_{\varphi}$-invariance. Since (3.H1) is satisfied, the conclusion $\left(\mathrm{D}_{1}\right)$ of Theorem 4.1.1 holds.

Assertion ( $\mathrm{D}_{2}$.d) follows from the inclusion $\widetilde{G}(1) \subseteq G_{1}$, which we prove next.

Let $g \in \widetilde{G}(1)$; let $x, y \in I_{k} \cap J_{k(r)}$ and take the points $x_{0}=x, x_{1}, \ldots, x_{n}=$ $y$ such that $s_{i}([0,1]) \subseteq I_{k}$ for $i=1, \ldots, n$, where $s_{i}(t)=x_{i-1}+\left(x_{i}-x_{i-1}\right) t$ for $t \in[0,1]$. Then

$$
\begin{aligned}
\left|\ln g\left(x_{i}\right)-\ln g\left(x_{i-1}\right)\right| & =\left|\int_{0}^{1} \frac{d}{d t} \ln \left(g\left(s_{i}(t)\right)\right) d t\right| \\
& \leq \operatorname{Reg}(g)\left|x_{i}-x_{i-1}\right| \quad \text { for } i=1, \ldots, n .
\end{aligned}
$$

Summing up these inequalities and using $\left(4.2 . \mathrm{M}_{1} 2\right)$, one gets

$$
\frac{g(x)}{g(y)} \leq \exp \left(\operatorname{Reg}(g) C_{0}|x-y|\right) \quad \text { for any } x, y \in I_{k},
$$

which, in turn, implies (b) of Definition 4.1.3.

In Case (II) the following counterpart of Theorem 4.1.2 holds:

Theorem 4.3.8 ([Bu85a, Th.4.1]). Let a Markov map $\varphi$ satisfy either (B) (3.H3), (4.2.H8), and (4.3.H9); or 
(C) $\quad m(I)<\infty$ and (4.1.H6), (4.2.H8), and (4.3.H9).

Then the conclusion of Theorem 4.1.2 holds. In particular, assertion $\left(\mathrm{D}_{2} . \mathrm{c}\right)$ of that theorem holds for densities in $\widetilde{G}(1) \subseteq G_{1}$.

Finally, we also have in Case (II) the following counterpart of Theorem 4.1.3 (an extension to the case $m(I)=\infty$ of Proposition 3.1 of [Bu91a]):

THEOREM 4.3.9. Let a Markov map $\varphi$ satisfy either

$\left(\mathrm{B}_{1}\right) \quad$ (3.H3), (4.1. $\left.\mathrm{H}_{1} 3\right),(4.2 . \mathrm{H} 8)$, and (4.3.H9); or

$\left(\mathrm{C}_{1}\right) \quad m(I)<\infty,\left(4.1 . \mathrm{H}_{1} 3\right),(4.1 . \mathrm{H} 6),(4.2 . \mathrm{H} 8)$, and (4.3.H9).

Then the conclusion of Theorem 4.1.1 holds. In particular, assertion $\left(\mathrm{D}_{2} . \mathrm{c}\right)$ of that theorem holds for densities in $\widetilde{G}(1) \subseteq G_{1}$.

P r o of. It follows from (4.4), (4.2.H8) and (4.3.H9) that for $r=1,2, \ldots$,

$$
\operatorname{Reg}\left(\sigma_{k(r)}\right) \leq \widetilde{C}_{5}:=C_{2} C_{1} /\left(C_{1}-1\right) .
$$

Next, by using $\left(4.2 . \mathrm{M}_{1} 2\right)$ one gets $(r=1,2, \ldots)$

$$
\sigma_{k(r)}(x) / \sigma_{k(r)}(y) \leq \exp \left(\operatorname{Reg}\left(\sigma_{k(r)}\right) C_{0}|x-y|\right)
$$

for each $J_{k(r)}$ and $x, y \in J_{k(r)}$.

The above two inequalities imply $\left(4 \cdot 1 \cdot \mathrm{H}_{1} 4\right)$ and $\left(4 \cdot 1 \cdot \mathrm{H}_{1} 5\right)$. Thus the assertion holds by Theorem 4.1.3.

In Remark 3.3 it was noted that conditions (3.H2), (3.H3), and (4.1.H6) ensure the recurrence property of the Markov maps considered. It was also remarked that (3.H2) is the most general. To justify this we give two examples. The first illustrates the fact that (3.H2) controls in a very effective way a possible tendency of the mass to escape to a fixed point under the action of the transformations in the bounded domain case.

EXAmPle 4.3.1 (see [Bu91a, Remark 3.2(b)]). For $k=1,2, \ldots$, let $I_{k} \subset$ $I=[0,1]$ be an open interval such that $m\left(I_{k}\right)=1 / 2^{k}$, and let $\varphi_{2 k-1}$ and $\varphi_{2 k}$ be linear transformations from $I_{2 k-1}$ onto $I$ and from $I_{2 k}$ onto $I_{2 k} \cup$ $I_{2 k+1}$, respectively. Clearly, the transformation defined, for $x \in \bigcup_{k=1}^{\infty} I_{k}$, by $\varphi(x)=\varphi_{k}(x)$ iff $x \in I_{k}$ is an expanding Markov map which does not satisfy (4.1.H6). Moreover, it does not satisfy (3.H3): Indeed, for $k=1,2, \ldots$, let $\widetilde{g}_{k}:=g_{(1, \ldots, 1,2 k)}$ be the density defined by (3.2) which corresponds to the multi-index $(1, \ldots, 1,2 k)$ of length $s$. Then $\widetilde{g}_{k} \geq(2 / 3)^{s} \widetilde{\sigma}_{(2 k, \ldots, 2 k)}$, where $\widetilde{\sigma}_{(2 k, \ldots, 2 k)}$ is the density defined by (3.3) corresponding to the $s$-multi-index $(2 k, \ldots, 2 k)$. It follows that

$$
d_{s n} \geq \sup _{k \geq 1} \int_{I \backslash V_{n}} \widetilde{g}_{k} d m \geq(2 / 3)^{s} \sup _{k \geq 1} \int_{I \backslash V_{n}} \widetilde{\sigma}_{(2 k, \ldots, 2 k)} d m=(2 / 3)^{s}
$$


for $s \geq 1$, where $d_{s n}$ is defined by (3.4). Note that here the densities $\widetilde{\sigma}_{(2 k, \ldots, 2 k)}$ $=(2 / 3) 2^{2 k} \mathbf{1}_{I_{2 k} \cup I_{2 k+1}}, k=1,2, \ldots$, are unbounded and with disjoint supports. However, the Markov map under consideration satisfies (3.H2) for $\widetilde{r}=1$, because

$$
g_{k} \geq \int_{A} \widetilde{\sigma}_{k} d m \text { and } \quad \int_{A} \widetilde{\sigma}_{2 k} d m \geq \frac{1}{3} \quad \text { where } \quad A=\bigcup_{i=1}^{\infty} I_{2 i-1},
$$

and $g_{k}$ is the density defined by (3.2) corresponding to $\widetilde{r}=1$.

It may also happen, in the unbounded domain case, that the mass tends to escape to infinity under the action of the transformations. In such cases (3.H3) may be no longer effective while (3.H2) may still hold. Such a behavior of a Markov map is illustrated by the following example.

EXAMPle 4.3.2 (see [Bu91, Example 2.1]). Let $\varphi_{0}$ be a twice differentiable function from $I_{0}=(-1,1)$ onto $\mathbb{R}$ such that $\left|D \varphi_{0}\right| \geq C_{1}>1$ and $\left|D^{2} \varphi_{0}\right| /\left(D \varphi_{0}\right)^{2} \leq C_{2}<\infty$. Let $\varphi_{2 k}(x)=\varphi_{0}(x-4 k)$ if $4 k-1<x<4 k+1$, and $\varphi_{2 k+1}(x)=\varphi_{0}(x-2(2 k+1))+2(2 k+1)$ if $4 k+1<x<4 k+3$ for $k=0, \pm 1, \pm 2, \ldots$ Then, for each $x \in \widetilde{I}=\bigcup_{k=-\infty}^{\infty} I_{k}$, put $\varphi(x)=\varphi_{k}(x)$ iff $x \in I_{k}=(2 k-1,2 k+1)$.

Since $\sigma_{2 k+1}(x)=\sigma_{0}(x-2(2 k+1)), \varphi$ does not satisfy (3.H3). Nevertheless, $\varphi$ does satisfy (3.H2) because

$$
g_{s} \geq \frac{1}{4} \sigma_{0} \int_{A} \sigma_{1} d m
$$

where $g_{s}$ is defined by (3.2) and $A$ is the union of all $I_{2 k}$ 's.

REMARK 4.3.1. In connection with the above two examples we note that (3.H2) ensures very effectively the required recurrence property. For example, in [JGB94] an example is given of transformations with invariant measure for which the conditions given in [Ry83] are not conclusive. However, none of these two results is decisive in the above first example. The conditions given in [JGB94] are conclusive neither in the bounded interval case (first example) nor in the unbounded interval case (second example). Note that one of the assumptions given there is (3.H3) (see condition (9) there).

5. Bernoulli property of some $C^{1+\alpha}$ Markov maps. In [Bu93] it is proved that some $C^{2}$ Markov maps have the Bernoulli property, that is, their natural extensions are isomorphic to Bernoulli shifts. In this section that property is extended to the $C^{1+\alpha}$ Markov maps of Theorem 4.1.3.

The following subclass of $G_{\alpha}$ (Definition 4.1.3) plays an important role in our further considerations: 
Definition 5.5. We denote by $G_{0, \alpha}, 0<\alpha \leq 1$, the family of all densities $g \in G$ satisfying the following three conditions:

(a) $\operatorname{spt}(g)$ is the union of a number of $I_{k}$ 's;

(b) for each $I_{k} \in \pi, g_{\mid I_{k}} \in C^{0+\alpha}\left(I_{k}\right)$, and

$|g(x)-g(y)| \leq C_{10}\left(C_{*} C_{* *}^{2}+1\right) g(y)|x-y|^{\alpha} \quad$ for all $x, y \in \operatorname{spt}(g) \cap I_{k} ;$

(c) $\int_{I \backslash V_{n}} g d m \leq C_{*} C_{* *} d_{\widetilde{r} n}$ for some $\widetilde{r}$ and all $n=1,2, \ldots$, where $d_{\widetilde{r} n}$ is defined by (3.4).

The lemma below gives two properties of this family of densities needed for the proof of the main result of this section, Theorem 5.1 and Corollary 5.1:

Lemma 5.1. $G_{0, \alpha}$ has the following properties:

(a) $\left\{P^{r} g_{0, k(r)}: k(r) \in K^{r}, r \geq \widetilde{r}\right\} \subseteq G_{0, \alpha}$, where

$$
g_{0, k(r)}=\mathbf{1}_{I_{k(r)}} g_{0} / \mu_{0}\left(I_{k(r)}\right),
$$

and $g_{0}$ is the unique $P_{\varphi}$-invariant density of Theorem 4.1.3;

(b) $\lim _{j \rightarrow \infty} a(j)=0$, where $a(j)=\sup \left\{\left\|P^{j} g-g_{0}\right\|: g \in G_{0, \alpha}\right\}$.

Pro of. (a) Since

$$
P^{r} g_{0, k(r)}(x)= \begin{cases}g_{0} \circ \varphi_{k(r)}^{-1}(x) \sigma_{k(r)}(x) / \mu_{0}\left(I_{k(r)}\right) & \text { for } x \in J_{k(r)}, \\ 0 & \text { for } x \in I \backslash J_{k(r)},\end{cases}
$$

one gets in particular $\operatorname{spt}\left(P^{r} g_{0, k(r)}\right)=J_{k(r)}$.

Next, it follows from inequality (4.3) (inserting $w_{k}=1_{I_{k}} / m\left(I_{k}\right) \in G_{\alpha}$ ) and Theorem 4.1.3 that for each $I_{k}$,

$$
\left|g_{0}(x)-g_{0}(y)\right| \leq C_{10} g_{0}(y)|x-y|^{\alpha} \quad \text { for all } x, y \in I_{k} .
$$

Using (5.1), the last inequality and (4.1.H4) one gets

$$
\left|P^{r} g_{0, k(r)}(x)-P^{r} g_{0, k(r)}(y)\right| \leq C_{10}\left(P^{r} g_{0, k(r)}(x)+P^{r} g_{0, k(r)}(y)\right)|x-y|^{\alpha} .
$$

Further, by Theorem 3.1(b) and (4.1.H5) one gets

$$
\sup _{I_{k}} g_{0} \leq C_{*} C_{* *} \inf _{I_{k}} g_{0} .
$$

From this, using once more (5.1), and then (4.1.H4) one gets

$$
\sup _{J_{k(r)} \cap I_{k}} P^{r} g_{0, k(r)} \leq C_{*} C_{* *}^{2} \inf _{J_{k(r)} \cap I_{k}} P^{r} g_{0, k(r)} .
$$

The last inequality and (5.2) imply that $P^{r} g_{0, k(r)}$ satisfies condition (b) of Definition 5.5. 
To show that $P^{r} g_{0, k(r)}$ also satisfies condition (c) of Definition 5.5 note first that

$$
\mu_{0}\left(I_{k(r)}\right)=\int_{I_{k(r)}} g_{0} d m \geq \inf _{I_{k(r)}} g_{0} \int_{J_{k(r)}} \sigma_{k(r)} d m
$$

From this, (5.1) and (5.3) it follows that

$$
P^{r} g_{0, k(r)}(x) \leq C_{*} C_{* *} \widetilde{\sigma}_{k(r)} .
$$

Finally, $P^{r} g_{0, k(r)}$ satisfies condition (c) of Definition 5.5 by the last inequality and Step 4.1.2.

(b) Note first that $G_{0, \alpha}$ is compact in the topology of uniform convergence (on each $I_{k}$ ). Indeed, it follows from Definition 5.5(b) that

$$
\sup _{\operatorname{spt}(g) \cap I_{k}} g \leq C_{* *, 1} \inf _{\operatorname{spt}(g) \cap I_{k}} g
$$

where $C_{* *, 1}:=1+C_{10}\left(C_{*} C_{* *}^{2}+1\right) \widetilde{C}_{0}^{\alpha}$ and $\widetilde{C}_{0}=\sup \left\{\operatorname{diam}\left(I_{k}\right): k \in K\right\}<\infty$. In particular, $\sup _{\operatorname{spt}(g) \cap I_{k}} g \leq C_{* *} / m\left(I_{k}\right)$.

Together with condition (b) of Definition 5.5 and the Ascoli-Arzelà Lemma, this implies the above compactness property of $G_{0, \alpha}$. This property, Definition 5.5(c), and the inequality (for $g \in G_{0, \alpha}$ )

$$
\sup _{\operatorname{spt}(g) \cap V_{n}} g \leq C_{* *, 1} / \widetilde{m}\left(V_{n}\right), \quad \text { where } \widetilde{m}\left(V_{n}\right)=\min \left\{m\left(I_{k}\right): I_{k} \subseteq V_{n}\right\},
$$

imply the compactness of $G_{0, \alpha}$ in $L^{1}$.

Finally, set $a(j, g):=\left\|P^{j} g-g_{0}\right\|$ for $g \in G_{0, \alpha}$. The sequence $j \rightarrow a(j, \cdot)$ is a pointwise decreasing sequence of continuous functionals defined on the compact set $G_{0, \alpha}$, and $\lim _{j \rightarrow \infty} a(j, g)=0$ for each $g \in G$ by Theorem 4.1.3. Thus (b) follows from Dini's Theorem.

Let $\left(I, \Sigma(I), \mu_{0} ; \varphi\right)$ be the semi-dynamical system generated by a Markov map $\varphi$ which satisfies the assumptions of Theorem 4.1.3. We now show that its natural extension is isomorphic to a Bernoulli shift.

To begin with we recall that the natural extension of $\left(I, \Sigma(I), \mu_{0} ; \varphi\right)$ is a quadruple $\left(\widetilde{I}, \Sigma(\widetilde{I}), \widetilde{\mu}_{0} ; \widetilde{\varphi}\right)$ whose elements can be defined as follows [Ro61]:

$$
\begin{gathered}
\widetilde{I}:=\left\{y=\left(y_{0}, y_{1}, \ldots\right) \in \prod_{j=0}^{\infty} I: \varphi\left(y_{j}\right)=y_{j-1} \text { for each } j \geq 1\right\} ; \\
\Sigma(\widetilde{I}):=\sigma\left(Y_{j}^{-1} \Sigma(I): j \geq 0\right),
\end{gathered}
$$

where

$$
Y_{j}: \widetilde{I} \rightarrow I \text { is the } j \text { th projection; }
$$


the countably additive measure $\widetilde{\mu}_{0}$ is first defined by

$$
\widetilde{\mu}_{0}\left(Y_{j}^{-1}(A)\right):=\mu_{0}(A) \quad \text { for } Y_{j}^{-1}(A) \in \bigcup_{i=0}^{\infty} Y_{j}^{-1} \Sigma(I),
$$

and then extended, by the Carathéodory-Hopf Theorem, to all of $\Sigma(\widetilde{I})$.

Finally, $\widetilde{\varphi}: \widetilde{I} \rightarrow \widetilde{I}$ is defined by

$$
\widetilde{\varphi}(y):=\left(\varphi\left(y_{j}\right)\right)=\left(\varphi\left(y_{0}\right), y_{0}, y_{1}, y_{2}, \ldots\right) \quad \text { for } y=\left(y_{0}, y_{1}, y_{2}, \ldots\right) .
$$

From the definition of $\widetilde{\varphi}$ it follows that it is one-to-one, and that it preserves the measure $\widetilde{\mu}_{0}$. The inverse of $\widetilde{\varphi}$ is given by

$$
\widetilde{\varphi}^{-1}(y)=\left(y_{1}, y_{2}, \ldots\right) \quad \text { for } y=\left(y_{0}, y_{1}, y_{2}, \ldots\right) .
$$

Further, it follows from the definition that

$$
\sigma\left(\bigcup_{j=-\infty}^{\infty} \widetilde{\varphi}^{j} Y_{0}^{-1} \Sigma(I)\right)=\Sigma(\widetilde{I})
$$

i.e. the $\sigma$-algebras $\widetilde{\varphi}^{j} Y_{0}^{-1} \Sigma(I), j=0, \pm 1, \pm 2, \ldots$, generate $\Sigma(\widetilde{I})$.

Actually, there exists a generator for $\widetilde{\varphi}$ :

LEMMA 5.2. Under the assumptions of Theorem 4.1 .3 the partition of $\widetilde{I}$ given by $\widetilde{\pi}:=Y_{0}^{-1} \pi$ is a generator for $\widetilde{\varphi}$, i.e. $\sigma\left(\bigcup_{j=-\infty}^{\infty} \widetilde{\varphi}^{j} \widetilde{\pi}\right)=\Sigma(\widetilde{I})$.

Proof. It follows from (5.7), (5.9), and (5.10) that

$$
\widetilde{\varphi}^{-j} \widetilde{\pi}=Y_{n}^{-1}\left(\varphi^{-(j+n)} \pi\right) \quad \text { for } n \geq 0 \quad \text { and } \quad j=-n,-n+1, \ldots
$$

Put $\mathcal{A}:=\sigma\left(\widetilde{\varphi}^{j} \widetilde{\pi}: j=0, \pm 1, \pm 2, \ldots\right)$. Then it follows that

$$
Y_{n}^{-1} \pi^{r+n}=\bigvee_{j=-(r+1)}^{n} \widetilde{\varphi}^{j} \widetilde{\pi} \subseteq \mathcal{A} \quad \text { for } n \geq 0 \text { and } r \geq 0 .
$$

This together with (4.1.H7) implies

$$
Y_{n}^{-1} \Sigma(I)=\sigma\left(\bigvee_{j=-(r+1)}^{n} \widetilde{\varphi}^{j} \widetilde{\pi}: r \geq 0\right) \subseteq \mathcal{A} \quad \text { for } n=0,1,2, \ldots
$$

From this and (5.6) it follows that $\mathcal{A}=\Sigma(\widetilde{I})$.

We recall that a partition $\varrho$ is independent if the $\sigma$-algebras $\sigma\left(\widetilde{\varphi}^{j} \varrho\right)$, $j=0, \pm 1, \pm 2, \ldots$, are independent, i.e. for any choice of distinct $j_{1}, \ldots, j_{r}$ and (not necessarily distinct) $U_{j_{1}}, \ldots, U_{j_{r}} \in \varrho$,

$$
\widetilde{\mu}_{0}\left(\widetilde{\varphi}^{-j_{1}} U_{j_{1}} \cap \ldots \cap \widetilde{\varphi}^{-j_{r}} U_{j_{r}}\right)=\widetilde{\mu}_{0}\left(U_{j_{1}}\right) \ldots \widetilde{\mu}_{0}\left(U_{j_{r}}\right) .
$$

Clearly, if $\widetilde{\pi}$ were an independent generator for $\widetilde{\varphi}$, then $\widetilde{\varphi}$ would be isomorphic to a Bernoulli shift. Note that in that case the entropy would be $h(\widetilde{\varphi})=h(\widetilde{\pi}, \widetilde{\varphi})=$ the entropy of the Bernoulli shift. 
There is a remarkable strengthening of that fact, in which the hypothesis of the independence of a generator is replaced by a sort of asymptotic independence ([FO70, IMT71, M71]).

Given two partitions $\varrho_{1}$ and $\varrho_{2}$ of $\widetilde{I}$ we define

$$
D\left(\varrho_{1}, \varrho_{2}\right)=\sum_{U \in \varrho_{1}} \sum_{V \in \varrho_{2}}\left|\widetilde{\mu}_{0}(U \cap V)-\widetilde{\mu}_{0}(U) \widetilde{\mu}_{0}(V)\right| .
$$

A partition $\varrho$ of $\widetilde{I}$ is said to be weak Bernoulli if

$$
\lim _{t \rightarrow \infty} D(t)=0,
$$

where

$$
D(t)=\sup _{r \geq 0} D\left(\bigvee_{j=-r}^{0} \widetilde{\varphi}^{j} \varrho, \bigvee_{j=t}^{t+r} \widetilde{\varphi}^{j} \varrho\right) .
$$

THEOREM 5.1. Under the assumptions of Theorem 4.1.3 the dynamical system $\left(\widetilde{I}, \Sigma(\widetilde{I}), \widetilde{\mu}_{0} ; \widetilde{\varphi}\right)$ is isomorphic to a Bernoulli shift.

Proof. The proof consists in checking (5.12). Let $\widetilde{V}=\widetilde{I}_{k_{0}} \cap \widetilde{\varphi}^{-1} \widetilde{I}_{k_{1}} \cap \ldots \cap$ $\widetilde{\varphi}^{-r} \widetilde{I}_{k_{r}} \in \bigvee_{j=-r}^{0} \widetilde{\varphi}^{j} \widetilde{\pi}$ and $\widetilde{U}=\widetilde{\varphi}^{t} \widetilde{I}_{\ell_{r}} \cap \widetilde{\varphi}^{t+1} \widetilde{I}_{\ell_{r-1}} \cap \ldots \cap \widetilde{\varphi}^{t+r} \widetilde{I}_{\ell_{0}} \in \bigvee_{j=t}^{t+r} \widetilde{\varphi}^{j} \widetilde{\pi}$ for $r=0,1,2 \ldots, t \geq 0$, where $\widetilde{I}_{k}=Y_{0}^{-1} I_{k}$ and $I_{k} \in \pi$.

Then from the $\widetilde{\varphi}$-invariance of $\widetilde{\mu}_{0}$, the relations

$$
\widetilde{\varphi}^{-1} Y_{0}^{-1}(A)=Y_{0}^{-1}\left(\varphi^{-j}(A)\right) \quad \text { for } j=0,1,2, \ldots,
$$

and (5.8), it follows that

$$
\widetilde{\mu}_{0}(\widetilde{V} \cap \widetilde{U})=\widetilde{\mu}_{0}\left(\widetilde{\varphi}^{-(t+r)}(\widetilde{V} \cap \widetilde{U})\right)=\mu_{0}(V \cap U),
$$

where $V=\varphi^{-(t+r)}\left(I_{k(r+1)}\right)$ and $U=I_{\ell(r+1)}$.

From (5.11) and (5.14) it follows that

$$
\begin{aligned}
& D\left(\bigvee_{j=-r}^{0} \widetilde{\varphi}^{j} \widetilde{\pi}, \bigvee_{j=t}^{t+r} \widetilde{\varphi}^{j} \tilde{\pi}\right)=\sum_{U} \sum_{V}\left|\mu_{0}(V \cap U)-\mu_{0}(V) \mu_{0}(U)\right| \\
& \quad=\sum_{U} \mu_{0}(U) \sum_{I_{k(r+1)}}\left|\int_{I_{k(r+1)}}\left\{P^{t-1}\left(P^{r+1} g_{0} \mathbf{1}_{U} / \mu_{0}(U)\right)-g_{0}\right\} d m\right| \\
& \quad \leq \sum_{U} \mu_{0}(U)\left\|P^{t-1} \widetilde{g}_{0, \ell(r+1)}-g_{0}\right\|,
\end{aligned}
$$

where $\widetilde{g}_{0, \ell(r+1)}=P^{r+1}\left(g_{0} \mathbf{1}_{U} / \mu_{0}(U)\right)$ and $U=I_{\ell(r+1)} \in \pi^{r+1}$.

This and Lemma 5.1(a) imply

$$
D\left(\bigvee_{j=-r}^{0} \widetilde{\varphi}^{j} \widetilde{\pi}, \bigvee_{j=t}^{t+r} \widetilde{\varphi}^{j} \widetilde{\pi}\right) \leq a(t-1) \quad \text { for } r \geq \widetilde{r}-1
$$


As for the remainder numbers, i.e. the numbers $(\widetilde{r}-1)-j(j=1, \ldots$, $\widetilde{r}-1)$, the density $P^{j} P^{\widetilde{r}-1-j} g_{0, k(\widetilde{r}-1-j)}$ is in $G_{0, \alpha}$ because it is a convex combination of densities in $G_{0, \alpha}$, and $G_{0, \alpha}$ is a convex set.

This case together with the previous one yields

$$
D(t) \leq \max \{a(t-\widetilde{r}+j): j=0,1, \ldots, \widetilde{r}-1\} \quad \text { for } t \geq \widetilde{r} .
$$

The last inequality and Lemma 5.1(b) show that the partition $\tilde{\pi}$ is weak Bernoulli. The proof is completed by an appeal to Lemma 5.2 and to [FO70, IMT71, M71].

In conclusion we show that the label-process $\left\{X_{t}\right\}_{t \geq 0}$ associated with the semi-dynamical system $\left(I, \Sigma(I) \mu_{0} ; \varphi\right)$, where $d \mu_{0}=g_{0} d m$, is absolutely regular, that is, $\lim _{t \rightarrow \infty} b(t)=0$, where $b(t)$ is defined by (5.16) below [IR78].

This process is defined over the probability space $\left(I, \Sigma, \mu_{0}\right)$ and takes values in $K$ (the index set of $\pi$ ) according to the following rule: $X_{t}=k$ iff $\varphi^{t}(x) \in I_{k}$.

For $t \geq 0, r \geq 1(t, r$ integers $)$, and $k(r+1)=\left(k_{0}, k_{1}, \ldots, k_{r}\right) \in K^{r+1}$, we define a cylindric set by

$$
A_{t} k(r+1)=\left\{X_{t}=k_{0}, X_{t+1}=k_{1}, \ldots, X_{t+r}=k_{r}\right\} .
$$

Note that $A_{t} k(r+1)=\varphi^{-t}\left(I_{k(r+1)}\right)$. Since $\mu_{0}$ is $\varphi$-invariant it follows that $\mu_{0}\left(A_{t} k(r+1)\right)=\mu_{0}\left(A_{0} k(r+1)\right)$. Hence the process in question is stationary.

For $0 \leq t \leq r<\infty$ we define $\Sigma_{t}^{t+r+1}=\sigma\left(A_{t}(r+1): k(r+1) \in K^{r+1}\right)$ and $\Sigma_{t}^{\infty}=\sigma\left(A_{t} k(r): k(r) \in K^{r}, r \geq 1\right)$. Note that $\Sigma_{t}^{t+r+1}=\sigma\left(\bigvee_{j=t}^{r} \varphi^{-j}(\pi)\right)$.

Then we define

$$
b(t)=\sup _{r \geq 1} E\left(\sup _{V \in \Sigma_{r+t}^{\infty}}\left|\mu_{0}\left(V \mid \Sigma_{0}^{r+1}\right)-\mu_{0}(V)\right|\right) .
$$

COROLlary 5.1. Under the assumptions of Theorem 4.1.3 the labelprocess $\left\{X_{t}\right\}_{t \geq 0}$ associated with the semi-dynamical system $\left(I, \Sigma(I) \mu_{0} ; \varphi\right)$, where $d \mu_{0}=g_{0} d m$, is absolutely regular, that is, $\lim _{t \rightarrow \infty} b(t)=0$.

Proof. For any $V \in \Sigma_{r+t}^{\infty}$ and $U \in \Sigma_{0}^{r+1}$ we have

$$
\begin{aligned}
\sup _{V} \mid \mu_{0}\left(V \mid \Sigma_{0}^{r+1}\right) & -\mu_{0}(V) \mid \\
& =\sup _{V}\left|\sum_{U} \mu_{0}(U)^{-1}\left(\int_{U} \mathbf{1}_{V} d \mu_{0}\right) \mathbf{1}_{U}-\mu_{0}(V)\right| \\
& =\sum_{U} \sup _{V}\left|\int_{V}\left\{\left(g_{0} \mathbf{1}_{U} / \mu_{0}(U)\right)-g_{0}\right\} d m\right| \mathbf{1}_{U} \\
& =\sum_{U} \sup _{\widetilde{V}}\left|\int \mathbf{1}_{\widetilde{V}}\left\{P^{t-1}\left(\widetilde{g}_{0, \ell(r+1)}\right)-g_{0}\right\} d m\right| \mathbf{1}_{U},
\end{aligned}
$$

where $V=\varphi^{-(r+t)}(\widetilde{V})$ for some $\widetilde{V} \in \Sigma_{0}^{\infty}$. 
From this and (5.16) we get (see the derivation of $\left(5.15^{*}\right)$ )

$$
b(t) \leq \max \{a(t-\widetilde{r}+j): j=0,1, \ldots, \widetilde{r}-1\} \quad \text { for } t \geq \widetilde{r} .
$$

The proof is completed by an appeal to Lemma 5.1(b).

REMARK 5.1. We note that $D(t) \leq 2 b(t)$. Indeed, from $\left(5.16^{*}\right)$ and the Hahn Decomposition Theorem we get

$$
E \sup _{V}\left|\mu_{0}\left(V \mid \Sigma_{0}^{r+1}\right)-\mu_{0}(V)\right|=\sum_{U} \mu_{0}(U)\left\|\left(P^{t-1} \widetilde{g}_{0, \ell(r+1)}-g_{0}\right)^{+}\right\| .
$$

On the other hand, by (5.15), we have

$$
D\left(\bigvee_{j=-r}^{0} \widetilde{\varphi}^{j} \widetilde{\pi}, \bigvee_{j=t}^{t+r} \widetilde{\varphi}^{j} \widetilde{\pi}\right) \leq \sum_{U} \mu_{0}(U)\left\|P^{t-1} \widetilde{g}_{0, \ell(r+1)}-g_{0}\right\|,
$$

and the assertion follows from (5.13) and (5.16).

6. Some special cases. There is a series of results in the literature connected with the theorems of Section 4. Some of them are incorrect, and there is a common reason of their incorrectness. We intend to elucidate that problem separately elsewhere. Here we only illustrate it with one case already published in [Bu85, Bu87]. This case is also connected with some corrected cases which occurred recently in the literature. In this section we give a few explanations and complements to them.

As already noted, (4.2.H8) and (4.3.H9) (more generally, Rényi's Conditions (4.1.H5) or (4.1. $\widetilde{H} 5)$ ) ensure the needed recurrence property of a smooth (say, $C^{2}$ ) Markov map $\varphi$ only under the following very special conditions (see Remarks 3.2 and 3.3):

$$
m(I)<\infty,
$$

and the Markov partition $\pi$ of $\varphi$ satisfies

$$
\pi \text { is finite or }\left(3 . \mathrm{M}_{1} 4\right) \text { holds. }
$$

The reason why this is the case is nowadays clear. Namely, under (6.1) and (6.2), $\varphi$ automatically satisfies (4.1.H6) given in Fact 4.1.1. Such a special case $\left((6.1)\right.$ and $\left.\left(3 . \mathrm{M}_{1} 4\right)\right)$ was considered, for instance, in [Re57, Ro61, A75, Sch89], and more recently $((6.1)$ and $\# \pi<\infty)$, in [A91; Folklore Theorem in Sect. 4.4.5] and [AF91; Folklore Theorem and Theorem B1(Folklore)].

During the past two decades or so, some attempts have been made to relax (in the case $m(I)<\infty$ ) the strongly restrictive alternative (6.2).

In [Bow79; "Adler's Theorem", p. 1], (6.2) was replaced by the following condition (see condition (d) of the definition of Markov map on p. 1 there):

$\left(6 . \mathrm{M}_{2} 4\right) \quad$ there exists $j \geq 1$ such that $I_{i} \subset \bigcup_{r=1}^{j} \varphi^{r}\left(I_{k}\right)$ for every $I_{i}, I_{k} \in \pi$. 
In [A79] an opinion was expressed that such a fact holds. The fact itself was called "the folklore theorem" there. (The meaning of "the folklore theorem" was then changed without any explanations in [A91] and [AF91].) On the other hand, the fact was questioned in [Se79]. Since no proof was given in [Bow79], the question arose whether such a general case holds.

A final answer was published in [Bu85, Bu87] where also conditions (3.H3) and (4.1.H6) were proposed as additional assumptions, while $\left(6 . \mathrm{M}_{2} 4\right)$ was replaced by (2.M4).

For the sake of completeness we present below one counterexample from the two papers:

Counterexample 6.1 ([Bu85, Bu87]). First, we take a stochastic matrix $M=\left[m_{i j}\right]_{i, j=1}^{\infty}$ with the following elements:

If $i=1$, then

$$
m_{i j}= \begin{cases}1-c & \text { if } j=1, \\ c 2^{-(j-1)} & \text { if } j=2,3, \ldots,\end{cases}
$$

and if $i=2,3, \ldots$, then

$$
m_{i j}= \begin{cases}c 2^{-2(i-2)(i-1)} 2^{-4 j} & \text { if } j=1, \ldots, i-1, \\ 1-c_{i} & \text { if } j=i, \\ c 2^{-(j-i)} & \text { if } j=i+1, i+2, \ldots\end{cases}
$$

where $0<c \leq 1 / 2$ and $c_{i}=c+\sum_{j=1}^{i-1} m_{i j}$.

Next, we associate with $M$ a piecewise linear transformation $\tau: I \rightarrow I$ $(I=[0, \infty))$ as follows. Let $I_{i j}=\left[a_{i j}, a_{i, j+1}\right)$ for $i=1,2, \ldots$, where

$$
a_{i j}=(i-1)+\sum_{k=1}^{j-1} m_{i k} \quad \text { if } j=2,3, \ldots, \quad a_{i 1}=i-1 .
$$

Let $\tau_{i j}$ be a linear mapping (increasing or decreasing) from $I_{i j}$ onto $I_{j}=[j-1, j)$. We define $\tau: I \rightarrow I$ by $\tau(x)=\tau_{i j}(x)$ iff $x \in I_{i j}$.

Then we define an auxiliary piecewise linear transformation $\lambda: I \rightarrow \widetilde{I}$ from $I=[0, \infty)$ onto $\widetilde{I}=[0,1]$ by

$$
\lambda(x)=\lambda_{i}(x) \quad \text { iff } \quad x \in I_{i}=[i-1, i), i=1,2, \ldots,
$$

where $\lambda_{i}: I_{i} \rightarrow \widetilde{I}_{i}$ is a linear mapping (e.g., increasing) from $I_{i}$ onto $\widetilde{I}_{i}=$ $\left[1-2^{-(i-1)}, 1-2^{-i}\right), i=1,2, \ldots$

Note that $\lambda_{i}\left(I_{i j}\right)=\widetilde{I}_{i j}=\left[\widetilde{a}_{i j}, \widetilde{a}_{i, j+1}\right)$ where

$$
\widetilde{a}_{i j}=\left(1-2^{-(i-1)}\right)+2^{-i} \sum_{k=1}^{j-1} m_{i k} \quad \text { if } \quad j=2,3, \ldots, \widetilde{a}_{i 1}=1-2^{-(i-1)} .
$$

Finally, we define the desired transformation $\widetilde{\tau}: \widetilde{I} \rightarrow \widetilde{I}$ by $\widetilde{\tau}=\lambda \circ \tau \circ \lambda^{-1}$. It is a Markov map with respect to the Markov partition $\widetilde{\pi}=\left\{\widetilde{I}_{i j}: i, j=\right.$ 
$1,2, \ldots\}$. Since $C_{1}^{-1}=\sup _{i, j \geq 1} 2^{-i} m_{i j} 2^{j}=1-c<1$, it satisfies (4.2.H8). Clearly, it satisfies (4.3. $\widetilde{\mathrm{H}} 9)$ but not (4.1.H6).

It turns out that $\widetilde{\tau}$ satisfies $\left(6 . \mathrm{M}_{2} 4\right)$ for the smallest possible $j=2$ (for $j=1$ we get $\left(3 . \mathrm{M}_{1} 4\right)$, and then the alternative (6.2) holds). Indeed,

$$
\widetilde{\tau}^{2}\left(\widetilde{I}_{i j}\right)=\widetilde{\tau}\left(\widetilde{I}_{j}\right)=\widetilde{\tau}\left(\bigcup_{i=1}^{\infty} \widetilde{I}_{j i}\right)=[0,1) \quad \text { for } i, j=1,2, \ldots
$$

Nevertheless there is no $\widetilde{\tau}$-invariant density. This is so because

$$
\left\|\mathbf{1}_{[j-1, j)} P_{\tau}^{i} g_{1}\right\| \leq \widetilde{m}\left(q_{s}^{i}+\frac{Q_{s}}{1-q_{s}}\right)+\widetilde{m}_{s, j} \quad \text { for } j, s=1,2, \ldots,
$$

where

$$
\begin{gathered}
g_{1}=\sum_{j=1}^{\infty} m_{1 j} \mathbf{1}_{[j-1, j)}, \quad \widetilde{m}=\sup _{i, j \geq 1} m_{i j}, \quad q_{s}=\sum_{j=1}^{s} m_{1 j} \leq \varepsilon<1, \\
Q_{s}=\sum_{j=1}^{s} m_{s+1, j} \leq(c / 15) 2^{-2 s(s-1)}, \quad \frac{Q_{s}}{1-q_{s}} \leq(1 / 15) 2^{(-2 s+1)(s-1)},
\end{gathered}
$$

and if $s \geq j$, then

$$
\widetilde{m}_{s j}=\sup _{i \geq 1} m_{s+i, j}=c 2^{-(2(s-1) s+4 j)} \rightarrow 0 \quad \text { as } s \rightarrow \infty .
$$

Several authors tried to replace the condition $\# \pi<\infty$ in (6.2) by the following one (see e.g. [P80, Th.1], [BowS79], or $\left.\left[\mathrm{S} 79,\left(d^{\prime \prime}\right)\right]\right)$ :

(6. $\left.\mathrm{H}_{1} 6\right) \quad \#\left\{\varphi^{j}(\partial U): j=1,2, \ldots ; U \in \pi\right\}<\infty$, where $\partial U=\operatorname{cl} U \backslash \operatorname{int} U$.

In [Bu82a] conditions (4.1.H6) for $m(I)<\infty$ and (3.H2) were given. Somewhat later in [Ry83] the case $\# \pi=\infty, m(I)<\infty$ was treated for not necessarily Markov maps. The conditions established there were complementary to those established for Markov maps (see Example 4.3.1 and Remark 4.3.1).

A review of some of the results connected with this topic is given in [I87] and [IG91, Sect. 5.3].

More recently, some results appeared (with $m(I)<\infty$ ) which include the indispensable condition (4.1.H6): [ADU93, Lem. 2.1], [MS93, Th. V.2.2], and quite recently $[\mathrm{Br} 94$, (e) in Sect. 4] and [Br94a, Folklore Theorem (Introduction), and (e) in 1.3]. Note that the origin of that condition was overlooked by all these authors.

Moreover, in [MS93, Remark 4c, p. 354] the authors gave an opinion on the role of (4.1.H6) (it is condition (c) in Def. on p. 353 of their book) for the existence of an invariant density, and on the possibility of its generalization. On the other hand, it was also overlooked by them that (3.H2) (an essential 
generalization of (4.1.H6), see Ex. 4.3.1) was already exploited, for instance, in [Bu91, Bu91a].

Condition (4.1.H6) plays a special role in [Br94, Br94a]. Namely, it is used there for determining a class of unimodal Markov maps called long-branched maps there (the condition itself is called "long-branchedness").

Note also that the "Folklore Theorem" used there differs from its original version given in [A79, A91, AF91]. Namely, in [A91, AF91] under the assumptions of the "Folklore Theorem" condition (4.1.H6) is automatically satisfied, while in [Br94, Bru94a] it is added to the hypotheses.

Theorem V.2.2 of [MS93] consists of two parts. The first part deals with reducible Markov maps (with (2.M4) dropped), while the second one deals with the irreducible ones. Note that in [MS93] condition (4.1.H6) is included in the definition of the Markov map ((c), p. 353). The reducible Markov maps are also considered in [ADU93, K90, Bu86] (see also [Bu92]). The irreducible case, under more general conditions (including the case $m(I)=\infty$ ), was already considered in [Bu82a] (see Remark 3.3) and in [Bu85, Bu85a, Bu91].

The proof of the second part of Theorem V.2.2 of [MS93] has a gap. Namely, a contradiction is derived from the following inequality ([MS93, p. 359]):

$$
\frac{m\left(\varphi^{r}\left(I_{k(r+1)}\right)\right)}{m\left(I_{k(r+1)}\right)} \geq \frac{1}{K} \frac{m\left(A_{r}\right)}{m\left(A \cap I_{k(r+1)}\right)},
$$

where $A=\varphi^{-r}\left(A_{r}\right)$, with $0<\mu(A)<1$, and Lebesgue's theorem on density points. If (6.3) holds, then the contradiction follows, provided

$$
\lim _{r \rightarrow \infty} m\left(I_{k(r)}\right)=0 \quad \text { for } I_{k(r+1)} \subset I_{k(r)}, r=1,2, \ldots
$$

There are, however, no comments on whether (6.4) holds. That question is handled in a very special case in [H77, Th. 5.2] $\left(C^{2}\right.$-smoothness, among other things, is assumed, while in [MS93] only $\left.C^{1+\alpha}\right)$. Note that in [Br94a, Sect. 1.3] it is noted (without giving any details) that (2.M4), (4.1.H5), and (4.1.H6) (resp., (d), (b), (e) in Section 1.3 there) imply (4.2.H8) (the latter yields the sufficient inequality in Fact 4.2.3).

On the other hand, the fact that $\pi$ is a generator for $\varphi$ (4.1.H7) is included in the definition of a (Markov) fibred system in [ADU93, Sect. 2].

Actually both (6.4) and (4.1.H7) can be proved under general, purely measure-theoretic assumptions (no smoothness assumptions are needed). Namely, the following two facts hold:

Proposition 6.1. Assume that:

(6. $\widetilde{\mathrm{H}} 1) \quad G^{*}\left(C^{*}\right) \neq \emptyset$ for some $C^{*}>0$, where $G^{*}$ is defined by (3.0); and

(a) for all $g \in G^{*}$, the limit $\lim _{j \rightarrow \infty} S_{j} g$, where $S_{j}=j^{-1} \sum_{i=0}^{j-1} P^{i}$, exists in $L^{1}$. 
Then

(b) $\quad \lim _{r \rightarrow \infty} m\left(I_{k(r)}\right)=0 \quad$ if $I_{k(r+1)} \subseteq I_{k(r)} \neq \emptyset$ for $r=1,2, \ldots$

Lemma 6.1. Let $I$ be a metric space, $\Sigma$ the $\sigma$-algebra of Borel sets in $I$, and $m$ a regular Borel measure on $I$. Suppose that for every sequence $\left\{I_{k(r)}\right\}_{r=1}^{\infty}$ of subsets of I given by (2.4), and satisfying $I_{k(r+1)} \subseteq I_{k(r)}$ $\neq \emptyset$, there exists a constant $C^{* *}=C^{* *}\left(\left\{I_{k(r)}\right\}\right)$ such that ess $\operatorname{diam}\left(I_{k(r)}\right)$ $\leq C^{* *} m\left(I_{k(r)}\right), r=1,2, \ldots$ Finally, assume that assertion (b) of the above proposition holds. Then (4.1.H7) holds, i.e., $\pi$ is a generator for $\varphi$.

The proofs will be given elsewhere.

\section{References}

[A75] R. L. Adler, Continued fractions and Bernoulli trials, Lecture notes, Ergodic Theory, J. Moser, E. Phillips, and S. Varadhan (eds.), Courant Inst. Math. Sci., New York, 1975.

[A79] —, Afterword (to [Bow79]), Comm. Math. Phys. 69 (1979), 15-17.

[A91] -, Geodesic flows, interval maps, and symbolic dynamics, in: Ergodic Theory, Symbolic Dynamics and Hyperbolic Spaces, T. Bedford, M. Keane and C. Series (eds.), Oxford Univ. Press, Oxford, 1991, 93-123.

[ADU93] J. Aaronson, M. Denker and M. Urbański, Ergodic theory for Markov fibred systems and parabolic rational maps, Trans. Amer. Math. Soc. 337 (1993), 495-548.

[AF91] R. L. Adler and L. Flatto, Geodesic flows, interval maps, and symbolic dynamics, Bull. Amer. Math. Soc. 25 (1991), 229-334.

[Bow79] R. Bowen, Invariant measures for Markov maps of the interval, Comm. Math. Phys. 69 (1979), 1-14.

[BowS79] R. Bowen and C. Series, Markov maps associated with Fuchsian groups, Inst. Hautes Études Sci. Publ. Math. 50 (1979), 401-418.

[Br94] H. Bruin, Topological conditions for the existence of invariant measures for unimodal maps, Ergodic Theory Dynam. Systems 14 (1994), 433-451.

[Br94a] -, Invariant measures of interval maps, Thesis, Technische Universiteit Delft 1994.

[Bu81] P. Bugiel, Notes on invariant measures for Markov maps of the interval, preprint, Jagiellonian University, 1981.

[Bu82] -, Approximation for the measures of ergodic transformations of the real line, Z. Wahrsch. Verw. Gebiete 59 (1982), 27-38.

[Bu82a] -, Ergodic properties of maps of Markov type $\left(\right.$ in $\left.\mathbb{R}^{1}\right)$, Thesis, Jagiellonian University, Kraków, 1982.

[Bu85] -, A note on invariant measures for Markov maps of an interval, Z. Wahrsch. Verw. Gebiete 70 (1985), 345-349.

[Bu85a] - On a class of piecewise monotonic transformations admitting smooth invariant measures, Prace Inf. Z.1, Zesz. Nauk. UJ, 726 (1985), 49-60.

[Bu86] - On the number of all absolutely continuous, ergodic measures of Markov type transformations defined on an interval, Math. Nachr. 129 (1986), 261268. 
[Bu87] P. Bugiel, Correction and addendum to "A note on invariant measures for Markov maps of an interval." Z. Wahrsch. Verw. Gebiete 70, 345-349 (1985), Probab. Theory Related Fields 76 (1987), 255-256.

[Bu91] -, On the convergence of iterates of the Frobenius-Perron operator associated with a Markov map defined on an interval. The lower-function approach, Ann. Polon. Math. 53 (1991), 131-137.

[Bu91a] —, Ergodic properties of Markov maps in $\mathbb{R}^{d}$, Probab. Theory Related Fields 88 (1991), 483-496.

[Bu92] - Invariant measures of $C^{2}$-diffeomorphisms of Markov type in $\mathbb{R}^{d}$, Monatsh. Math. 113 (1992), 255-263.

[Bu93] - On a Bernoulli property of some piecewise $C^{2}$-diffeomorphisms in $\mathbb{R}^{d}$, ibid. 116 (1993), 99-110.

[Bu96] - Application of the Ulam's procedure for approximating invariant measures to the case of $C^{1+\alpha}$-smooth Markov maps in $\mathbb{R}^{d}$, Univ. Iagell. Acta Math. 34 (1997), 7-27.

[DS63] N. Dunford and J. T. Schwartz, Linear Operators, Part I, General Theory, Wiley, New York, 1963.

[FO70] N. Friedman and D. Ornstein, On isomorphism of weak Bernoulli transformations, Adv. Math. 5 (1970), 365-394.

[G69] G. M. Goluzin, Geometric Theory of Functions of a Complex Variable, Transl. Math. Monographs 26, Amer. Math. Soc., 1969.

[H77] M. Halfant, Analytic properties of Rényi's invariant density, Israel J. Math. 27 (1977), 1-20.

[IMT71] S. Ito, H. Murata and H. Totoki, Remarks on the isomorphism theorems for weak Bernoulli transformations in general case, Publ. Res. Inst. Math. Sci. 7 (1971/72), 541-580.

[IR78] I. A. Ibragimov and Ya. A. Rozanov, Gaussian Random Processes, Springer, Berlin, 1978.

[I87] M. Iosifescu, Mixing properties for f-expansions, in: Probab. Theory and Math. Statist., Vol. II, Yu. V. Prohorov et al. (eds.), VNU Science Press, 1987, 1-8.

[IG91] M. Iosifescu and S. Grigorescu, Dependence with Complete Connections and its Applications, Cambridge Tracts in Math. 96, Cambridge Univ. Press, Cambridge, 1990.

[JGB94] M. Jabłoński, P. Góra and A. Boyarsky, A general existence theorem for absolutely continuous invariant measures on bounded and unbounded intervals, preprint, 1994, to appear.

[K90] G. Keller, Exponents, attractors and Hopf decompositions for interval maps, Ergodic Theory Dynam. Systems 10 (1990), 717-744.

[LY82] A. Lasota and J. Yorke, Exact dynamical systems and the Frobenius-Perron operator, Trans. Amer. Math. Soc. 273 (1982), 375-384.

[Li71] M. Lin, Mixing for Markov operators, Z. Wahrsch. Verw. Gebiete 16 (1971), 231-242.

[M71] G. Maruyama, Some aspects of Ornstein's theory of isomorphism problems in ergodic theory, Publ. Res. Inst. Math. Sci. 7 (1971/72), 511-539.

[Ma87] R. Mañé, Ergodic Theory and Differentiable Dynamics, Ergeb. Math. Kuznzgeb. 8, Springer, Berlin, 1987.

[MS93] W. de Melo and S. van Strien, One-Dimensional Dynamics, Ergeb. Math. Grenzgeb. 25, Springer, Berlin, 1993. 
[P80] G. Pianigiani, First return map and invariant measures, Israel J. Math. 35 (1980), 32-47.

[R56] O. Rechard, Invariant measures for many-one transformations, Duke Math. J. 23 (1956), 477-488.

[Re57] A. Rényi, Representations for real numbers and their ergodic properties, Acta Math. Acad. Sci. Hungar. 8 (1957), 477-493.

[Ro61] V. A. Rokhlin, Exact endomorphisms of Lebesgue spaces, Izv. Akad. Nauk SSSR Ser. Mat. 25 (1961), 490-530 (in Russian); English transl.: Amer. Math. Soc. Transl. (2) 39 (1964), 1-36.

[Ry83] M. R. Rychlik, Bounded variation and invariant measures, Studia Math. 76 (1983), 69-80.

[Sch89] F. Schweiger, Ergodic theory of fibred systems, Institut für Mathematik der Universität Salzburg, Salzburg, 1989.

[Se79] C. Series, Additional comments (to [Bow79]), Comm. Math. Phys. 69 (1979), 17.

[Sz84] W. Szlenk, An Introduction to the Theory of Smooth Dynamical Systems, PWN, Warszawa, 1984.

[U60] S. M. Ulam, A Collection of Mathematical Problems, Interscience Tracts in Pure and Appl. Math., Interscience, New York, 1960.

Institute of Mathematics and Computer Science

Jagiellonian University

Nawojki 11

30-072 Kraków, Poland

E-mail: uibugiel@cyf-kr.edu.pl

Reçu par la Rédaction le 13.1.1997

Révisé le 20.5.1997 